\title{
Bulk and element-specific magnetism of medium-entropy and high-entropy Cantor-Wu alloys
}

\author{
D. Billington $\odot,{ }^{1,2,3, *}$ A. D. N. James $\odot,{ }^{1}$ E. I. Harris-Lee $\odot,{ }^{1}$ D. A. Lagos $\odot,{ }^{1}$ D. O’Neill $\odot,{ }^{4}$ N. Tsuda, ${ }^{3}$ K. Toyoki, \\ Y. Kotani $\odot,{ }^{3}$ T. Nakamura, ${ }^{3}$ H. Bei $\odot,{ }^{5}$ S. Mu $\odot,{ }^{5}$ G. D. Samolyuk, ${ }^{5}$ G. M. Stocks, ${ }^{5}$ J. A. Duffy, ${ }^{4}$ J. W. Taylor, ${ }^{6}$ \\ S. R. Giblin, ${ }^{2}$ and S. B. Dugdale (1) ${ }^{1}$ \\ ${ }^{1}$ H. H. Wills Physics Laboratory, University of Bristol, Tyndall Avenue, Bristol BS8 1TL, United Kingdom \\ ${ }^{2}$ School of Physics and Astronomy, Cardiff University, Queen's Building, The Parade, Cardiff CF24 3AA, United Kingdom \\ ${ }^{3}$ Japan Synchrotron Radiation Research Institute, SPring-8, Sayo 679-5198, Japan \\ ${ }^{4}$ Department of Physics, University of Warwick, Coventry CV4 7AL, United Kingdom \\ ${ }^{5}$ Materials Science and Technology Division, Oak Ridge National Laboratory, Oak Ridge, Tennessee 37831, USA \\ ${ }^{6}$ DMSC - European Spallation Source, Universitetsparken 1, Copenhagen 2100, Denmark
}

(Received 14 August 2020; accepted 12 October 2020; published 5 November 2020)

\begin{abstract}
Magnetic Compton scattering, x-ray magnetic circular dichroism spectroscopy, and bulk magnetometry measurements are performed on a set of medium- (NiFeCo and $\mathrm{NiFeCoCr}$ ) and high-entropy $(\mathrm{NiFeCoCrPd}$ and $\mathrm{NiFeCoCrMn}$ ) Cantor-Wu alloys. The bulk spin momentum densities determined by magnetic Compton scattering are remarkably isotropic, and this is a consequence of the smearing of the electronic structure by disorder scattering of the electron quasiparticles. Nonzero x-ray magnetic circular dichroism signals are observed for every element in every alloy indicating differences in the populations of the majority and minority spin states implying finite magnetic moments. When $\mathrm{Cr}$ is included in the solid solution, the $\mathrm{Cr}$ spin moment is unambiguously antiparallel to the total magnetic moment, while a vanishingly small magnetic moment is observed for $\mathrm{Mn}$, despite calculations indicating a large moment. Some significant discrepancies are observed between the experimental bulk and surface magnetic moments. Despite the lack of quantitative agreement, the element-specific surface magnetic moments seem to be qualitatively reasonable.
\end{abstract}

DOI: 10.1103/PhysRevB.102.174405

\section{INTRODUCTION}

It has been known for centuries that combining elements together in an alloy can result in the material having superior properties. Traditionally, however, there would be one principal component (e.g., Fe) to which smaller amounts of other elements (e.g., C) are added. Indeed, the Bronze Age is characterized by the technological advance enabled by the discovery that adding small amounts of other elements (such as $\mathrm{Sn}$ ) to $\mathrm{Cu}$ produced a harder metal. Building on early work published mainly in undergraduate theses (see, for example, Ref. [1]), several publications [2-6] established in 2004 the existence of a new type of alloy that is formed not by adding small amounts of other elements to one principal component, but by combining several elements in approximately equiatomic proportions. These alloys are often referred to as "high-entropy alloys" (HEAs) [7-12], which was a term first coined by Yeh et al. who attributed the high configurational entropy as the mechanism stabilizing the solid solution phase [6]. The terms "multicomponent alloys" and "multiprincipal element alloys" are also commonly used.

HEAs have complete substitutional disorder, meaning that all of the component elements in the material randomly

\footnotetext{
*Present address: School of Physics and Astronomy Cardiff University, Queen's Building, The Parade, Cardiff CF24 3AA, United Kingdom; billingtond1@ cardiff.ac.uk
}

occupy the crystallographic sites and, as such, these alloys do not have long-range compositional order (although there is growing evidence that short-range order can exist in HEAs [9,11-17]). This degree of disorder introduces unusual and unexpected behavior including enhanced mechanical properties such as hardness and resistance to wear, tensile strength, ductility, and fracture resistance $[7,9-12,18]$. These enhanced mechanical properties have seen high-entropy alloys become candidate materials for potential next-generation engineering applications including use in state-of-the-art racing cars, spacecraft, submarines, jet aircraft, and nuclear reactors [19-21].

Shortly after Yeh named these alloys, Cantor et al. developed the prototypical, equiatomic high-entropy alloy $\mathrm{NiFeCoCrMn} \mathrm{[2],} \mathrm{known} \mathrm{as} \mathrm{the} \mathrm{"Cantor} \mathrm{alloy,"} \mathrm{which} \mathrm{has}$ been the subject of considerable work in the field. More recently, Wu et al. [22] showed that alloying the individual elements of Cantor's NiFeCoCrMn alloy with each other and with Pd produced a series of two-, three-, and four-component equiatomic fcc solid solutions collectively referred to as Cantor-Wu alloys [2,22] that include $\mathrm{NiPd}, \mathrm{NiCo}, \mathrm{NiFe}$, NiFeCo, NiCoCr, NiCoMn, NiCoCrMn, NiFeCoMn, $\mathrm{NiFeCoCr}, \mathrm{NiFeCoCrMn}$, and NiFeCoCrPd. Currently, there is not a universal classification system which exactly qualifies an alloy to be a HEA, but alloys with five or more elemental components with this high substitutional disorder are generally considered to be HEAs [7-13,23] while alloys containing few components are given the appellation "medium-entropy 
TABLE I. Previously reported experimental Curie temperatures, $T_{\mathrm{C}}$, spin freezing temperatures, $T_{\mathrm{f}}$, Kondo temperatures, $T_{\mathrm{K}}$, and saturated magnetic moments, $m^{\text {sat }}$, for selected Cantor-Wu alloys taken from Refs. [26-30,36].

\begin{tabular}{lcccc}
\hline \hline & & & & $\begin{array}{c}m^{\text {sat }} \\
\text { Alloy }\end{array}$ \\
\hline NiFeCo [26,36] & 995 & & & 1.7 \\
NiFeCoCrPd [26,30] & 440 & & & 0.52 \\
NiFeCoCr [26,28] & 120 & 35 & & 0.24 \\
NiCoCr [26,27] & $<2$ & & & 0 \\
NiFeCoCrMn [26,29] & 38 & 93 & 40 & $<0.01$ \\
\hline \hline
\end{tabular}

alloys." Although much of the interest in HEAs stems from their potential for use in industrial and technological applications, from a fundamental physics perspective the Cantor-Wu alloys display a rich variety of electronic and magnetic behavior.

The Cantor-Wu alloys represent a mixture of $3 d$ transitionmetal ions and it is well known that magnetism (specifically, the $d$-band filling) in the $3 d$ transition metals is responsible for both their particular ground-state crystal structures and their mechanical properties [24,25]. So far, experimental information regarding the magnetism comes from bulk magnetometry measurements [26-31] which only provide the species averaged total (the sum of spin and orbital) magnetic moments. The only available element-specific information about the magnetism comes from ab initio calculations [26,29,31-35]. The combination of bulk magnetometry measurements and calculations has revealed that most of the Cantor-Wu alloys studied here are either ferromagnetic or ferrimagnetic. NiFe$\mathrm{CoCr}$ and $\mathrm{NiFeCoCrMn}$ have also been reported to exhibit spin-glass behavior [26,28,29], and Kondo-like behavior has been observed in $\mathrm{NiFeCoCrMn}$ as evidenced by an upturn in the resistivity at low temperatures. Table I lists the previously reported experimental values of the Curie temperatures, $T_{\mathrm{C}}$, and (where relevant) spin freezing temperatures, $T_{\mathrm{f}}$, and Kondo temperatures, $T_{\mathrm{K}}$, together with their saturated magnetic moments, $m^{\text {sat }}$ [26-30]. Except for NiFeCoCrMn, the Curie temperatures and saturated magnetic moments decrease with increasing $\mathrm{Cr}$ concentration and it has been argued that this implies a $\mathrm{Cr}$ moment aligned antiparallel to the average total moment, as predicted by calculations [26,29,33-35].

The presence of strong compositional disorder and magnetism in these alloys results in nontrivial electronic transport properties. Residual resistivity measurements show that the Cantor-Wu alloys are split into two subgroups with low $(<10 \mu \Omega \mathrm{cm})$ and high $(>75 \mu \Omega \mathrm{cm})$ residual resistivities [26,37]. Interestingly, the members of these two groups are not determined by the number of component elements, but instead are determined by the type of elements present. Korringa-Kohn-Rostoker (KKR) calculations employing the coherent potential approximation (CPA), which can effectively treat the compositional disorder, indicate substantial smearing of the electronic structure due to scattering of the electron quasiparticles [33,34,37-39]. For alloys containing only $\mathrm{Fe}, \mathrm{Co}$, and/or $\mathrm{Ni}$, the majority spin channel experiences negligible disorder scattering, thereby providing a short circuit, while for $\mathrm{Cr}$ - and $\mathrm{Mn}$-containing alloys both spin channels experience strong disorder scattering due to an electron filling effect [33]. Very recently, it was found experimentally that $\mathrm{NiFeCoCr}$ has a quasiparticle coherence length that is very close to the nearest-neighbor interatomic distance [40], i.e., approaching the Mott-Ioffe-Regel limit where the standard picture of ballistically propagating quasiparticles becomes invalid [41]. In fact, NiCoCr, NiFeCoCrMn, and $\mathrm{NiFeCoCrPd}$ all have residual resistivities which are higher than $\mathrm{NiFeCoCr}$, and all exhibit non-Fermi liquid behavior [26] and should probably be classed as trivial non-Fermi liquids [42]. Quantum critical behavior has been reported in $\operatorname{NiCoCr}_{x}(x \approx 1)$, whose magnetic moment vanishes due to strong magnetic fluctuations [27]. Despite all of these observations, there is currently no element-specific experimental information about the magnetic moments of each alloy.

In this study, we report magnetic-field-dependent synchrotron x-ray experiments with circularly polarized photons and bulk magnetometry for a set of medium- (NiFeCo and $\mathrm{NiFeCoCr}$ ) and high-entropy (NiFeCoCrPd and $\mathrm{NiFe}-$ CoCrMn) Cantor-Wu alloys [2,22]. Magnetic Compton scattering $[43,44]$ is used to determine the magnetic Compton profiles (MCPs) along high-symmetry crystallographic directions. The MCPs are one-dimensional projections of the underlying three-dimensional bulk spin momentum density which is intimately related to the many-body ground-state electronic wavefunction. Magnetic Compton scattering can determine the bulk spin moment and can be used to determine the bulk orbital moment by subtracting the bulk spin moment from the total bulk moment determined, for example, by bulk magnetometry measurements. X-ray magnetic circular dichroism (XMCD) [45] spectroscopy at the $L_{2,3}$ edges of the $3 d$ elements $\left(M_{2,3}\right.$ edges of $\left.\mathrm{Pd}\right)$ is exploited to obtain element-specific orbital and spin magnetic moments via the orbital and spin sum rules $[46,47]$, and to track their variation with applied magnetic field.

Before proceeding, we would like to emphasize that we do not expect the XMCD orbital and spin sum rules to provide quantitatively accurate values for the spin and orbital moments of the Cantor-Wu alloys due to the inherent surface sensitivity of total electron yield detection, the maximal compositional disorder of the measured alloys, and uncertainties in quantities that enter the sum rule equations, such as the element-specific $d$-electron occupancy of each alloy and the expectation value of the magnetic dipole operator at the surface of the samples. Despite this, the XMCD spectra will unambiguously reveal whether there is a finite moment and whether it is aligned parallel or antiparallel to the applied field. Furthermore, we expect the relative sizes of the sum rule moments to be qualitatively correct.

\section{METHODS}

\section{A. Crystal growth}

Details of the single crystal growth can be found in Refs. [22,48]. Ingots of each alloy were produced by arcmelting the constituent elements in a water-cooled copper hearth, under an Ar atmosphere. The arc-melted buttons were flipped and remelted five times in order to improve the 
compositional homogeneity, before being drop cast into square cross-section copper molds. These polycrystalline ingots were then loaded into an optical floating zone furnace to produce a single crystal which was subsequently cut into a disk using electrodischarge machining before being electrolytically polished to remove any damage caused by the cutting.

\section{B. Sample preparation}

For the magnetic Compton scattering measurements, the samples were aligned along high-symmetry crystallographic directions using x-ray Laue diffraction. The fcc crystal symmetry was evident in the recorded diffraction patterns.

In order to remove the contaminated surface oxide layer for the $\mathrm{x}$-ray absorption measurements, all of the samples were chemically etched for $3 \mathrm{~min}$ in a solution of distilled $\mathrm{H}_{2} \mathrm{O}$, 37 wt $\% \mathrm{HCl}$, and 70 wt $\% \mathrm{HNO}_{3}$ with a $\mathrm{H}_{2} \mathrm{O}: \mathrm{HCl}: \mathrm{HNO}_{3}$ volume ratio of 1:2:1. After etching, the samples were introduced to the ultrahigh vacuum (UHV) chamber of the soft x-ray absorption spectrometer apparatus. The samples were then sputtered in situ with an Ar ion plasma. For the Ar ion sputtering, the acceleration voltage was $2 \mathrm{kV}$, the emission current was $10 \mathrm{~mA}$, the incident angle was $45^{\circ}$, the Ar pressure was $6.7 \times 10^{-3} \mathrm{~Pa}$, and the duration was $2 \mathrm{~h}$. There was no sample alignment for the $\mathrm{x}$-ray absorption measurements, partly due to the sample preparation procedure, but also because cubic systems necessarily have low magnetocrystalline anisotropy.

\section{Bulk magnetometry measurements}

The magnetic field dependence of the total bulk magnetic moments, $m_{z}^{\text {tot }}\left(H_{\text {ext }}\right)$, of NiFeCo, NiFeCoCr, NiFeCoCrPd, and $\mathrm{NiFeCoCrMn}$ were measured at $T=10 \mathrm{~K}$ using a superconducting quantum interference device (SQUID). All of the samples were cooled in zero field.

\section{Magnetic Compton scattering measurements}

The electron momentum density from Bloch electrons can be written as

$$
\begin{aligned}
\rho(\mathbf{p}) & =\sum_{j, \mathbf{k}} n_{\mathbf{k}, j}\left|\int \psi_{\mathbf{k}, j}(\mathbf{r}) \exp (-\mathbf{i p} \cdot \mathbf{r}) d^{3} \mathbf{r}\right|^{2} \\
& =\sum_{j, \mathbf{k}, \mathbf{G}} n_{\mathbf{k}, j}\left|a_{\mathbf{G}, j}(\mathbf{k})\right|^{2} \delta(\mathbf{p}-\mathbf{k}-\mathbf{G}),
\end{aligned}
$$

where $\psi_{\mathbf{k}, j}(\mathbf{r})$ is the real-space wavefunction of an electron in band $j$ with wave vector $\mathbf{k}, n_{\mathbf{k}, j}$ is its occupancy which takes values between zero (unoccupied) and one (fully occupied), and the $\delta$ function expresses the contribution from higher momentum (umklapp) components whose intensities are given by the Fourier coefficients of the real-space electron wavefunctions, $a_{\mathbf{G}, j}(\mathbf{k})$. This means that an occupied electron state will contribute to the electron momentum density not only at $\mathbf{p}=\mathbf{k}$ but also at $\mathbf{p}=\mathbf{k}+\mathbf{G}$, where $\mathbf{G}$ is any reciprocal lattice vector.

The Compton profile $(\mathrm{CP}), J\left(p_{z}\right)$, is defined as the onedimensional (twice-integrated) projection of the electron momentum density, $\rho(\mathbf{p})$, along the scattering vector which is parallel (orthogonal) to $p_{z}\left(p_{x, y}\right)$,

$$
J\left(p_{z}\right)=\iint \rho(\mathbf{p}) d p_{x} d p_{y},
$$

which is normalized to the number of electrons, $N$ :

$$
N=\int J\left(p_{z}\right) d p_{z}
$$

It is also possible to write the total electron momentum density as the sum of contributions from the momentum densities of electrons with spins aligned parallel, $\rho_{\uparrow}(\mathbf{p})$, or antiparallel, $\rho_{\downarrow}(\mathbf{p})$, to a chosen spin quantization axis (the scattering vector),

$$
\rho(\mathbf{p})=\rho_{\uparrow}(\mathbf{p})+\rho_{\downarrow}(\mathbf{p}),
$$

and we can then define the electron spin momentum density as

$$
\rho_{\text {spin }}(\mathbf{p})=\rho_{\uparrow}(\mathbf{p})-\rho_{\downarrow}(\mathbf{p}) .
$$

The MCP, $J_{\mathrm{mag}}\left(p_{z}\right)$, is defined as the one-dimensional (twiceintegrated) projection of the electron spin momentum density, $\rho_{\text {spin }}(\mathbf{p})$, along the scattering vector which is parallel (orthogonal) to $p_{z}\left(p_{x, y}\right)$,

$$
J_{\mathrm{mag}}\left(p_{z}\right)=\iint\left[\rho_{\uparrow}(\mathbf{p})-\rho_{\downarrow}(\mathbf{p})\right] d p_{x} d p_{y},
$$

which is normalized to the electron spin moment, $m_{z}^{\text {spin }}$,

$$
m_{z}^{\text {spin }}=\int J_{\text {mag }}\left(p_{z}\right) d p_{z} .
$$

Magnetic Compton scattering is only sensitive to the spin magnetic moment $[49,50]$. Because only those electrons that contribute to the spin moment of the sample contribute to the integral of the MCP, it is then possible to determine the spin magnetic moment, usually by comparison with a measurement, under the same experimental conditions, of a sample with known spin moment (in this case, fcc $\mathrm{Ni}$ ). Since the MCP is the difference between two measured CPs, components arising from spin-paired electrons cancel, as do most sources of systematic error.

In practice, the spin magnetic moment along the scattering vector is determined from the so-called flipping ratio, $R$, defined as

$$
R=\frac{I^{\uparrow}-I^{\downarrow}}{I^{\uparrow}+I^{\downarrow}}=\frac{\int J_{\mathrm{mag}}\left(p_{z}\right) d p_{z}}{\int J\left(p_{z}\right) d p_{z}}=\frac{m_{z}^{\mathrm{spin}}}{N},
$$

where $I^{\uparrow}$ and $I^{\downarrow}$ are the integrated intensities when the sample moment is aligned parallel and antiparallel to the (fixed) photon helicity. By comparing the flipping ratio of the sample in question with that of a calibration sample of known spin moment, $m_{\mathrm{cal}}^{\mathrm{spin}}$, the experimental spin moment of the sample in question is then given by

$$
m_{\text {expt }}^{\text {spin }}=\frac{R_{\text {expt }}}{R_{\text {cal }}} \frac{N_{\text {expt }}}{N_{\text {cal }}} m_{\text {cal }}^{\text {spin }},
$$

where $N_{\text {expt }}$ and $N_{\text {cal }}$ are the number of electrons in the experimental and calibration samples, respectively. Typically, the chosen calibration sample is fcc $\mathrm{Ni}$ with $m_{\text {cal }}^{\text {spin }}=$ $0.56 \mu_{\mathrm{B}}$ atom $^{-1}$. 
In this study, MCPs were measured along the cubic highsymmetry [100], [110], and [111] directions for NiFeCo, $\mathrm{NiFeCoCr}$, and $\mathrm{NiFeCoCrPd}$ on beamline BL08W at the SPring-8 synchrotron, Japan. All of the MCPs were recorded at $T=10 \mathrm{~K}$ and $\mu_{0} H_{\text {ext }}= \pm 2 \mathrm{~T}$. All of the samples were cooled in zero field. The experimental full-width-athalf-maximum (FWHM) resolution was about 0.45 a.u. at the Compton peak. The incident photon energy was 183.4 $\mathrm{keV}$. The measured profiles were then corrected for energydependent detector efficiency, sample absorption, the relativistic scattering cross section, and multiple scattering. The data analysis has been described in detail previously $[43,44]$.

\section{E. X-ray absorption measurements}

In x-ray absorption spectroscopy (XAS), the x-ray absorption spectrum is given by $\mu(E)=\left[\mu^{+}(E)+\mu^{-}(E)\right] / 2$ and the XMCD spectrum is given by $\Delta \mu(E)=\left[\mu^{+}(E)-\right.$ $\left.\mu^{-}(E)\right]$, where $\mu^{+}(E)$ and $\mu^{-}(E)$ represent the energydependent absorption cross sections of soft x-ray photons with positive and negative helicity, $h^{+}$and $h^{-}$, respectively. In practice, the energy is fixed for each data point at a chosen energy, $E$, and the absorption signal is given by

$$
\mu_{E}^{ \pm}=\int \mu^{ \pm}\left(E^{\prime}\right) \delta\left(E^{\prime}-E\right) d E^{\prime},
$$

where the $\delta$ function represents the chosen (Gaussian) energy resolution.

The absorption signals were recorded with the soft x-ray absorption spectrometer on BL25SU [51-54], at the SPring-8 synchrotron, Japan, by means of the total electron yield (TEY) method. This apparatus is equipped with an electromagnet with a maximum applied magnetic field of $\mu_{0} H_{\text {ext }}= \pm 1.9 \mathrm{~T}$, and a cryostat which can cool the sample down to $10 \mathrm{~K}$. In this experiment, the energy resolution was set to $E / \Delta E=3000$, where $\Delta E$ is the Gaussian FWHM. Soft XMCD spectroscopy using TEY detection is a surface sensitive magnetic probe which has a probing depth (exponential decay length) of about $1 \mathrm{~nm}$ from the sample surface [55-57]. For Cr, Mn, Fe, Co, and $\mathrm{Ni}$, the spectra were recorded across their respective $L_{2,3}$ edges ( $2 p \rightarrow 3 d$ transitions), while for Pd the spectra were recorded across its $M_{2,3}$ edges $(3 p \rightarrow 4 d$ transitions). All of the spectra were recorded at $T=10 \mathrm{~K}$ and $\mu_{0} H_{\text {ext }}= \pm 1.9 \mathrm{~T}$ in order to saturate the moments. The recorded spectra were normalized by the TEY intensity monitored with a SiC membrane located upstream of the sample.

\section{Orbital and spin sum rules}

Measurement of the XAS and XMCD spectra permits the determination of the $z$ component (along the X-ray incidence direction) of the orbital and spin magnetic moments via application of the orbital and spin sum rules [46,47]. The sum rules are expressed in terms of various integrals over the XAS and XMCD spectra that are given the symbols $p, q$, and $r$ [58]. For the $3 d$ elements, $p$ is the integral of the XMCD spectrum over only the $L_{3}$ edge, i.e., the integral ends at the onset of the $L_{2}$ edge,

$$
p=\int_{L_{3}} \Delta \mu(E) d E
$$

$q$ is the integral of the XMCD spectrum over both the $L_{2}$ and $L_{3}$ edges,

$$
q=\int_{L_{3}+L_{2}} \Delta \mu(E) d E,
$$

and $r$ is the integral of the background corrected XAS spectrum, $\mu^{0}(E)=\mu(E)-f_{\text {bkg }}(E)$, over both the $L_{2}$ and $L_{3}$ edges,

$$
r=2 \int_{L_{3}+L_{2}} \mu^{0}(E) d E
$$

where $f_{\mathrm{bkg}}(E)$ is the nonmagnetic background which is a quadratic constructed from the linear gradients of the pre$L_{3}$ - and post- $L_{2}$-edge regions of $\mu(E)$, and two arctan step functions (one centered at the $L_{2}$ edge and one centered at the $L_{3}$ edge) for the continuum absorption [59].

For the $3 d$ elements, the sum rules then state that the $z$ component of the orbital magnetic moment (in units of $\mu_{\mathrm{B}}$ atom $^{-1}$ ) is given by

$$
m_{z}^{\text {orb }}=\frac{-4 q\left(10-n_{3 d}\right)}{3 r P_{c} \cos (\alpha)},
$$

and the $z$ component of the effective spin magnetic moment (in units of $\mu_{\mathrm{B}}$ atom $^{-1}$ ) is given by

$$
m_{z}^{\text {spin,eff }}=m_{z}^{\text {spin }}-7\left\langle T_{z}\right\rangle=\frac{-(6 p-4 q)\left(10-n_{3 d}\right)}{r P_{c} \cos (\alpha)}
$$

where $m_{z}^{\text {spin }}=-2\left\langle S_{z}\right\rangle$ (in Hartree atomic units), $\left\langle S_{z}\right\rangle$ and $\left\langle T_{z}\right\rangle$ are the expectation values of the $z$ components of the spin angular momentum operator and magnetic dipole operator, respectively, $n_{3 d}$ is the site averaged number of $3 d$ electrons of the element in question (determined by electronic structure calculations), $P_{c}=0.96$ [52] is the degree of circular polarization, and $\alpha=10^{\circ}$ is the angle between the applied field direction and the incident x-ray direction [53]. Note that $\left\langle T_{z}\right\rangle$ is generally assumed to be negligible for atoms in a cubic environment $[47,60]$. For all of the measured alloys, the spin sum rule moments of $\mathrm{Cr}$ were doubled because, for $\mathrm{Cr}$, the spin-orbit splitting of the $2 p_{3 / 2}$ and $2 p_{1 / 2}$ core levels is small resulting in significant overlap of the $L_{3}$ and $L_{2}$ absorption edges and, hence, spin sum rule moments that are too small by about a factor of 2 [59]. Accordingly, the spin sum rule moments are expected to be more accurate for $\mathrm{Fe}, \mathrm{Co}$, and $\mathrm{Ni}$ because of the larger spin-orbit splitting.

\section{Element-specific hysteresis loops}

The variation of the total magnetic moment, $m_{z}^{\text {tot }}=m_{z}^{\text {orb }}+$ $m_{z}^{\text {spin }}$, with $H_{\text {ext }}$ is proportional to

$$
m_{z}^{\text {tot }}\left(H_{\mathrm{ext}}\right) \propto \frac{\Delta \mu_{L_{2}}\left(H_{\mathrm{ext}}\right)}{\mu_{L_{2}}\left(H_{\mathrm{ext}}\right)}-\frac{\Delta \mu_{L_{3}}\left(H_{\mathrm{ext}}\right)}{\mu_{L_{3}}\left(H_{\mathrm{ext}}\right)},
$$

where the on-edge XMCD signals are normalized by their respective XAS signals in order to account for the nonlinear variation of the TEY signal with $H_{\text {ext }}$ [61] (note that for $\mathrm{Pd}$, we replace $L_{2,3}$ with $M_{2,3}$ ). All of the hysteresis loops were recorded at $T=10 \mathrm{~K}$ between applied magnetic fields of $\mu_{0} H_{\mathrm{ext}}= \pm 1.9 \mathrm{~T}$. The measured element-specific hysteresis loops for $\mathrm{Cr}, \mathrm{Mn}, \mathrm{Fe}, \mathrm{Co}$, and $\mathrm{Ni}$ were scaled to equal their respective $m_{z}^{\text {tot }}$ as determined by the sum rule analysis of the 
TABLE II. Magnetic moments from the orbital and spin sum rules (XMCD), KKR-CPA calculations (KKR), and bulk (SQUID and Compton) measurements. The numbers in parentheses are statistical errors of one standard deviation at the magnitude of the least significant figure. The statistical errors in the values derived from the bulk measurements are dominated by the statistical error in the spin moment from Compton scattering so the errors in the total moments from the SQUID have been omitted. For NiFeCoCrPd, the average $m^{\text {orb }}$ and average $m^{\text {spin,eff }}$ from the sum rules were determined using their respective Pd $d$-electron moments from the KKR-CPA calculations. The Pd $d$-electron moments and those derived from them are indicated by asterisks.

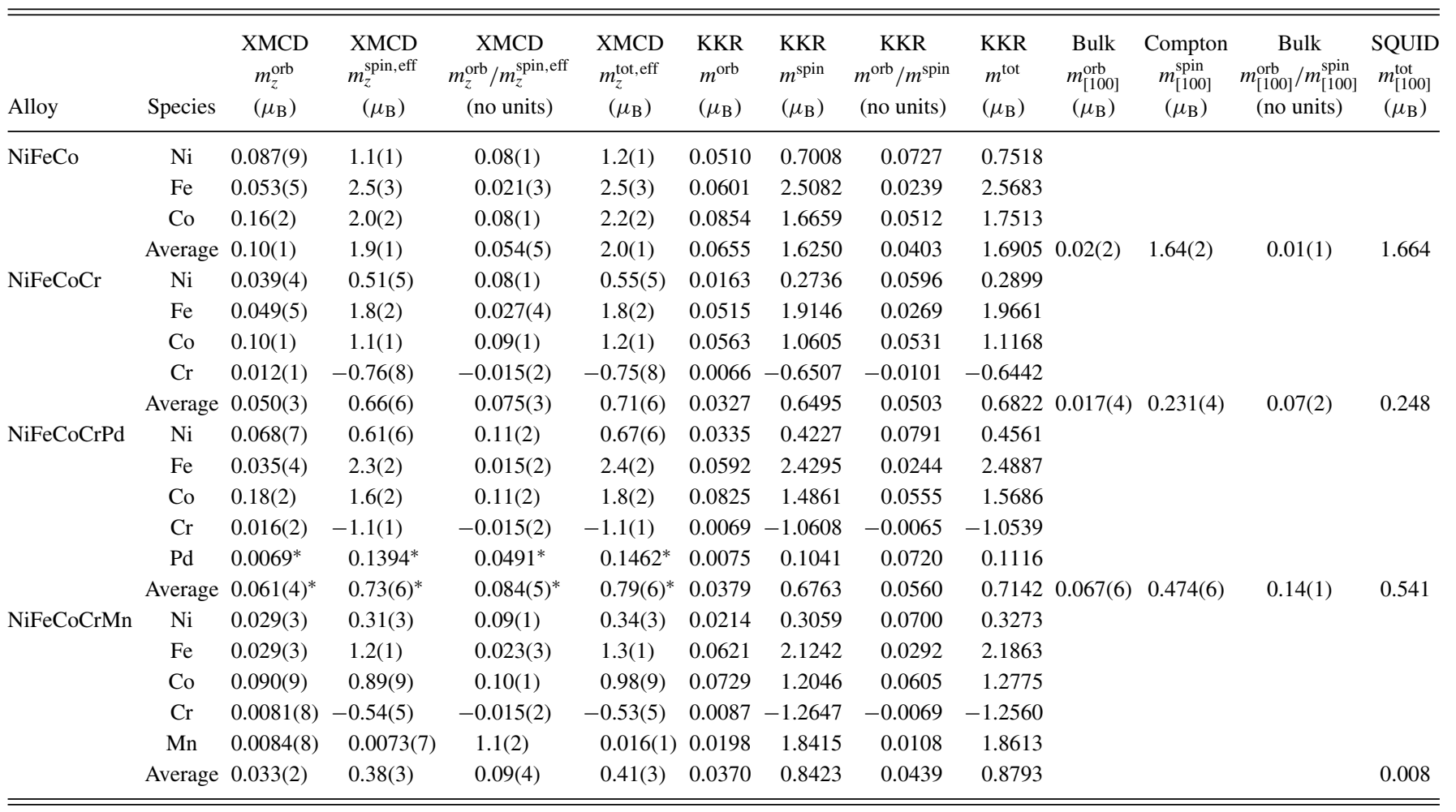

recorded spectra, while that of Pd was scaled to the total $\mathrm{Pd} d$ electron magnetic moment given by KKR-CPA calculations.

\section{F. Electronic structure calculations}

The KKR method [62-64] was used to calculate the electronic structure and the CPA [65-67] was used to treat the compositional disorder. The KKR-CPA calculations were performed with the Munich SPR-KKR code [68] within the atomic sphere approximation (ASA). The core configuration for all elements except Pd was $1 s^{2} 2 s^{2} 2 p^{6} 3 s^{2} 3 p^{6}$, and the core configuration for $\mathrm{Pd}$ was $1 s^{2} 2 s^{2} 2 p^{6} 3 s^{2} 3 p^{6} 3 d^{10} 4 s^{2} 4 p^{6}$. The lattice constants for $\mathrm{NiFeCo}, \mathrm{NiFeCoCr}$, and $\mathrm{NiFeCoCrMn}$ were $3.577 \AA$, while that of NiFeCoCrPd was $3.657 \AA$ due to the atomic size mismatch [30,69]. For NiFeCo, NiFeCoCr, and $\mathrm{NiFeCoCrMn}$, the muffin-tin radii were 2.38 a.u. while for $\mathrm{NiFeCoCrPd}$ the muffin-tin radii were 2.44 a.u. and $1200 k$ points were used to sample the irreducible wedge of the first Brillouin zone. The local density approximation (LDA) to the exchange-correlation energy functional was Vosko-WilkNusair (VWN) [70].

From the converged electronic structures, directional MCPs were calculated along the same directions as measured in the experiment. For comparison with the experimental MCPs, all of the calculated MCPs were convoluted with a Gaussian function with the FWHM equal to the experimental resolution at the Compton peak (0.45 a.u.).

\section{RESULTS}

The experimental and calculated MCPs of NiFeCo, NiFe$\mathrm{CoCr}, \mathrm{NiFeCoCrPd}$, and $\mathrm{Ni}$ (the $\mathrm{Ni}$ experimental data were previously reported in Ref. [71]) are shown in Figs. 1(a), 1(b), 1(c), and 1(d), respectively, resolved along the cubic [100], [110], and [111] high-symmetry crystallographic directions. The inset to each figure shows the anisotropy of the spin density in momentum space in the differences between MCPs measured along different crystallographic directions. The areas under the experimental profiles are equal to the experimental bulk spin moments [determined by Eq. (9)] and are listed in Table II. Superficially, the general shape of the MCPs of the Cantor-Wu alloys are similar to those of $\mathrm{Ni}$; they are finite at $p_{z}=0$ a.u. and rise to a broad maximum around $1<\left|p_{z}\right|<2$ a.u. with a tail that asymptotically approaches zero with increasing momentum.

The reduced intensity at low momentum in a MCP can be due to a number of factors. It is at low momentum that the most itinerant valence electrons are contributing to the momentum density. The rapid increase in intensity in a MCP could come from the negative net spin polarization of the delocalized (hence relatively localized in momentum space) $s p$ electrons that screen the more localized (hence delocalized in momentum space) $d$-electron moment (which has a much larger positive net spin polarization). Note that the degree of negative polarization of the $s p$ electrons in metallic $3 d$ moment systems is, typically, underestimated by LDA density 

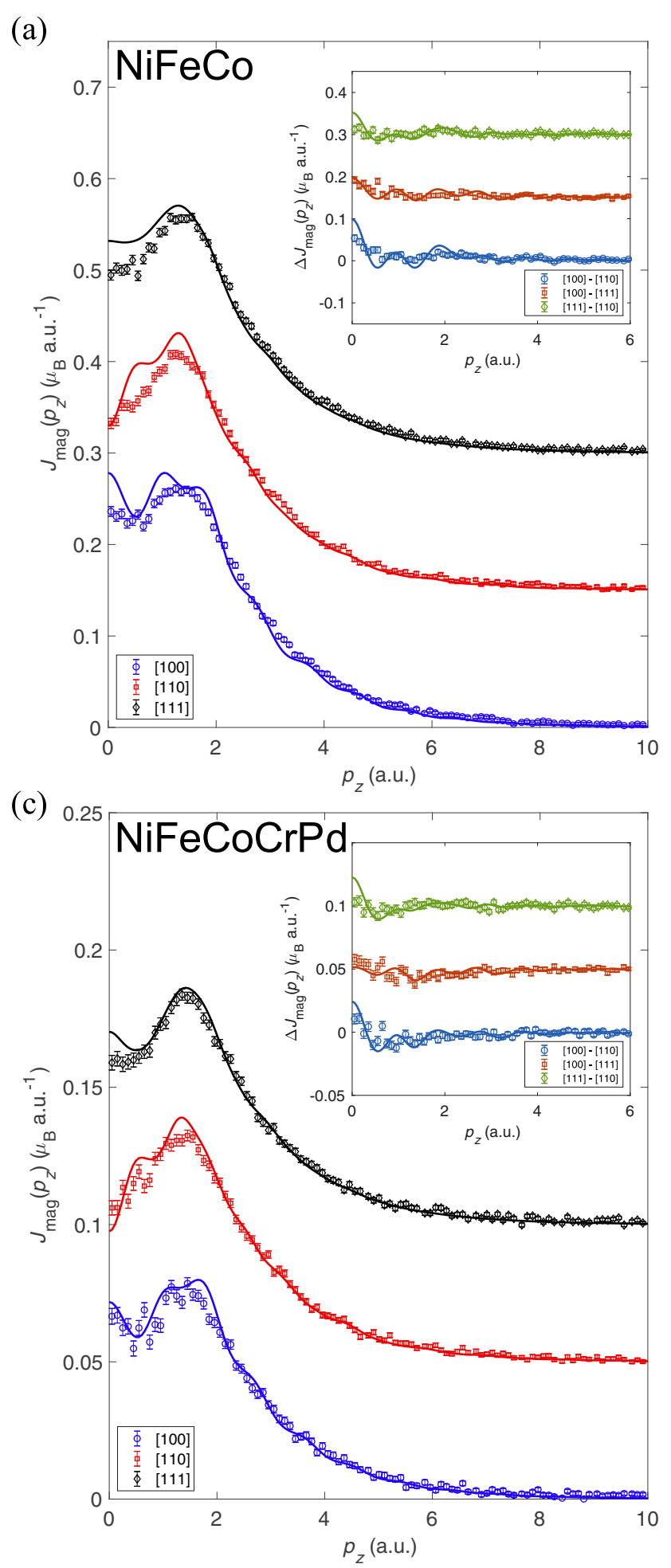

(b)

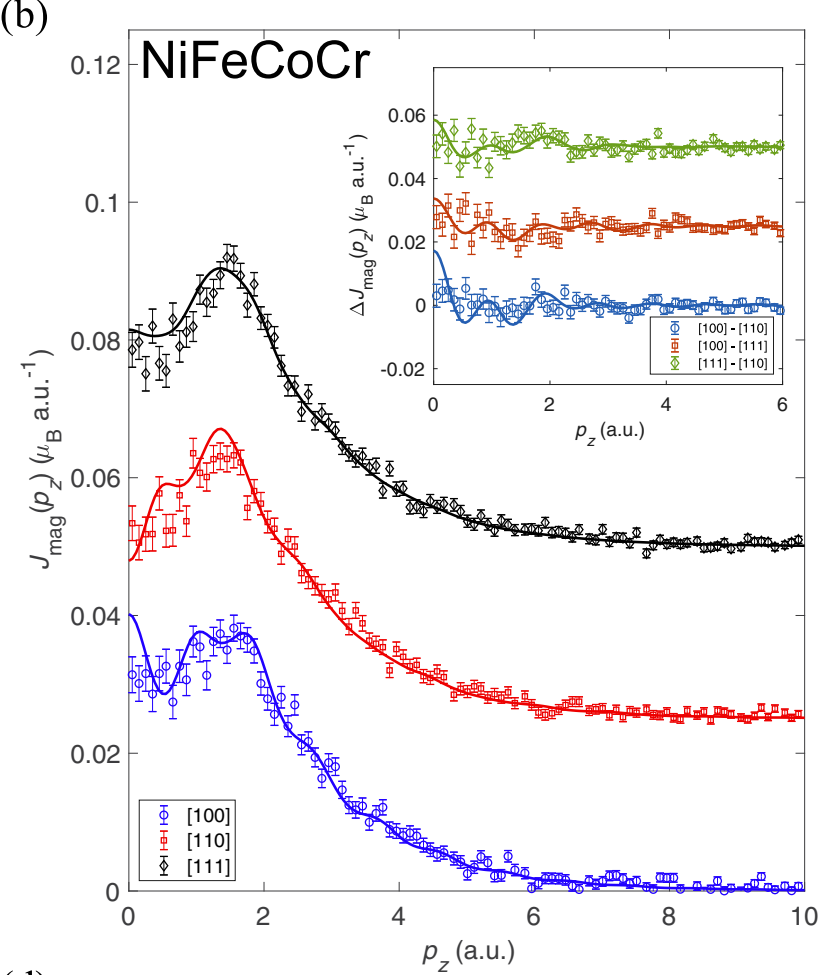

(d)

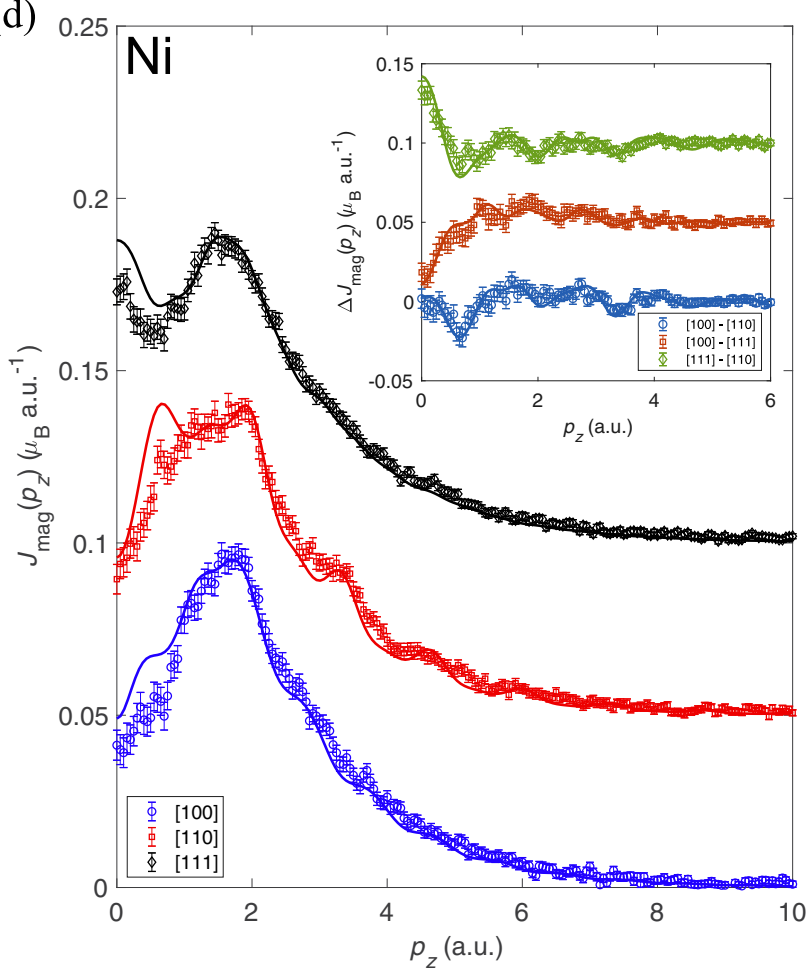

FIG. 1. Experimental (points) and calculated (lines) MCPs, $J_{\text {mag }}\left(p_{z}\right)$, of (a) NiFeCo, (b) NiFeCoCr, (c) $\mathrm{NiFeCoCrPd}$, and (d) fcc Ni recorded with the scattering vector parallel to the [100] (blue circles, bottom), [110] (red squares, middle), and [111] (black diamonds, top) high-symmetry crystallographic directions. The insets show the directional differences of the MCPs, $\Delta J_{\text {mag }}\left(p_{z}\right)$, between the [100] and [110] (blue circles, bottom), [100] and [111] (orange squares, middle), and [111] and [110] (green diamonds, top) directions. The directional profiles in each panel and directional differences in each inset have been offset by steps of (a) $0.15 \mu_{\mathrm{B}}$ a.u. ${ }^{-1}$, (b) $0.025 \mu_{\mathrm{B}}$ a.u. ${ }^{-1}$, (c) $0.05 \mu_{\mathrm{B}}$ a.u. ${ }^{-1}$, and (d) $0.05 \mu_{\mathrm{B}}$ a.u. ${ }^{-1}$ for clarity. The experimental and calculated directional profiles have been normalized to their experimentally determined bulk spin moments [from Eq. (9)] along their respective directions. The error bars are statistical errors of one standard deviation. Note that the error bars are larger when the bulk spin magnetic moment is smaller due to the measured signal being proportional to the spin magnetization. The fcc Ni experimental profiles are taken from Ref. [71]. 
functional theory (DFT) calculations [71-74]. It could also be due to the radial behavior of the wavefunction in momentum space which for a $d$-electron orbital goes like $p_{z}^{2}$ [75], resulting in a small contribution to the momentum density at low momentum which grows strongly as $p_{z}^{4}$. Finally, it could be a consequence of the Fermi surface, for example, through the presence of a majority hole pocket or minority electron pocket at the $\Gamma$ point, but this is not the case in these alloys. The disagreement at low momentum shows that the negative spin polarization is sensitive to the treatment of exchange and correlation [71]. Dynamical mean field theory has been investigated as a possible solution to this problem [76-78].

On closer inspection, however, it is clear that the MCPs of $\mathrm{Ni}$ show much more structure and anisotropy than those of the Cantor-Wu alloys, particularly for $\left|p_{z}\right|>2$ a.u. where higher momentum umklapp features are much more prominent. These features are due to the Fermi surface and arise from majority and minority bands crossing the Fermi energy at different crystal momenta (and different real momenta), leading to small peaks or troughs (for example, see the theoretical profiles in Ref. [71]). Although these sharp structures are smeared by the typical experimental resolution (the FWHM is about half of the size of the Brillouin zone), features such as shoulders (e.g., Ni [110] at $\left|p_{z}\right| \approx 3.5$ a.u.) can be resolved in the Ni data [Fig. 1(d)]. The MCPs of each CantorWu alloy are remarkably similar along each crystallographic direction and, for a given direction, the profile shapes are quite similar between the different Cantor-Wu alloys. In comparison to Ni, the MCPs of the Cantor-Wu alloys appear smeared. Robarts et al. very recently used high-resolution (nonmagnetic) Compton scattering to experimentally determine the bulk Fermi surface geometry of $\mathrm{NiFeCoCr}$, and found that it is smeared over $\sim 40 \%$ of the Brillouin zone [40]. Such smearing implies a short electron mean free path and thus a high residual resistivity. The Bloch spectral functions calculated by $\mathrm{Mu}$ et al. [33] show that the majority spin Fermi surface remains very sharp in $\mathrm{NiFeCo}$ (since the majority spin band centers align with each other for these $3 d$ elements), explaining the relatively low resistivity as the conductivity short circuits via the majority spin channel.

Focusing on the insets in Fig. 1, the intensities of the anisotropies are markedly smaller than the calculations predict for all of the Cantor-Wu alloys. For Ni [Fig. 1(d)], the agreement between the experiment and calculation is excellent. This implies that an inadequate DFT description (due to the use of Kohn-Sham wavefunctions [79] and/or inadequate exchange-correlation potential) is unlikely to be responsible for the overestimation of the anisotropy in the calculations for the Cantor-Wu alloys. Furthermore, it indicates that, whatever inadequacies there are in the calculation for $\mathrm{Ni}$, they disappear in the double difference between spins and crystallographic directions. This anisotropy is not observably smaller in $\mathrm{NiFeCo}$, where calculations suggest that only the minority Fermi surface is smeared [33]. The apparent "isotropy" of the MCPs of the Cantor-Wu alloys is, in fact, symptomatic of the smearing of the electronic structure by the compositional disorder. The high residual resistivity of Cr-containing alloys, being emblematic of a strongly smeared Fermi surface, suggests that the electrons are going to be ignorant of any phenomena involving coordination over distances much longer than their mean free path. Given that this distance is of the order of the lattice spacing, the lack of anisotropy in the momentum space spin density is perhaps not surprising.

The XAS and XMCD spectra of the measured absorption edges of NiFeCo and $\mathrm{NiFeCoCr}$ are shown in Fig. 2, and those of NiFeCoCrPd and NiFeCoCrMn are shown in Fig. 3. Note that the Pd XAS spectrum is not shown because the SiC membrane (whose TEY signal is used to normalize the measured spectra to the incident photon flux) has some adsorbed oxygen from exposure to air and the $\operatorname{Pd} M_{2,3}$ edges are in the same energy range as the $\mathrm{O} K$ edge, meaning that quantitative Pd moments cannot be determined. In all of the measured alloys, the $\mathrm{Ni}, \mathrm{Fe}$, and $\mathrm{Co}$ XAS and XMCD spectra have very similar shapes to that of their respective pure metals $[58,80]$ indicating undetectable levels of surface oxidation of these elements (the XAS of $\mathrm{Ni}, \mathrm{Fe}$, and $\mathrm{Co}$ oxides exhibits split peak structures at both the $L_{2}$ and $L_{3}$ edges due to multiplet effects; see, e.g., Refs. [81-83]). The relative sizes of the jumps in the XAS at the $L_{3}$ edge $\left(\mu_{L_{3}}-\mu_{\text {pre- } L_{3}}\right)$ of $\mathrm{Ni}, \mathrm{Fe}$, and Co (and $\mathrm{Cr}$ in the Cr-containing alloys) within an alloy are the same between different alloys, indicating that the relative chemical concentrations of these elements are not changing between the different alloys $[\mathrm{Ni}: \mathrm{Fe}: \mathrm{Co}(: \mathrm{Cr})$ is constant].

Nonzero XMCD signals are observed for every element in every alloy indicating finite magnetic moments, although for each element the sizes of the XMCD signals relative to their respective XAS signals vary significantly between alloys. Except for $\mathrm{Cr}$, the XMCD signals of all of the measured elements of each alloy are negative at the $L_{3}$ edge and positive at the $L_{2}$ edge (negative at the $M_{3}$ edge and positive at the $M_{2}$ edge for $\mathrm{Pd}$ ), indicating that the $z$ components of the site-averaged moments are parallel to the applied magnetic field and are ferromagnetically coupled to each other. In each Cr-containing alloy, the Cr XMCD signal is positive at the $L_{3}$ edge and negative at the $L_{2}$ edge, indicating that the $z$ component of the site-averaged moment is unambiguously aligned antiparallel to the applied magnetic field and that $\mathrm{Cr}$ is therefore antiferromagnetically coupled to the other elements in agreement with first-principles calculations [26,29,33-35]. For a quantitative determination of the element-specific orbital and spin magnetic moments, the orbital and spin sum rules [Eqs. (14) and (15), respectively] were applied to the measured spectra of $\mathrm{Ni}, \mathrm{Fe}, \mathrm{Co}, \mathrm{Cr}$, and $\mathrm{Mn}$. The values obtained are listed in Table II.

The $m_{z}^{\text {tot }}\left(H_{\text {ext }}\right)$ curves determined by Eq. (16) from the XAS and XMCD data are shown in Fig. 4, together with the bulk total moment curves obtained from the SQUID magnetometry measurements. NiFeCo has the largest moment, so would be most susceptible to demagnetization effects, although it is unclear how to correct for demagnetization effects at the sample surface. Nevertheless, the magnetic moments of all alloys are saturated at the maximum applied magnetic field of $\mu_{0} H_{\mathrm{ext}}= \pm 1.9 \mathrm{~T}$, which is where the XAS and XMCD spectra were recorded. The element-specific magnetization curves of the spin-glass alloys $\mathrm{NiFeCoCr}$ and $\mathrm{NiFeCoCrMn}$ exhibit hysteresis which is related to the energy barrier that must be overcome in order for the frozen magnetic moments to rearrange themselves. 
(a)

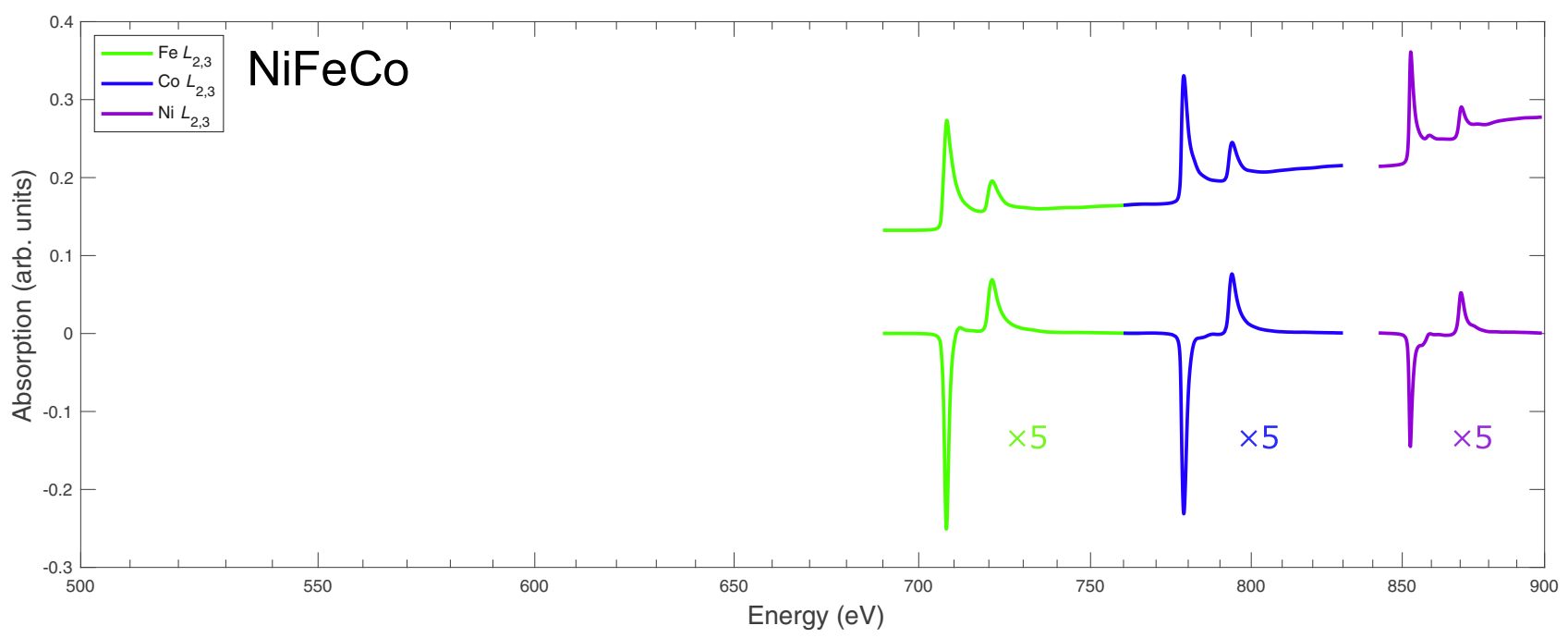

(b)

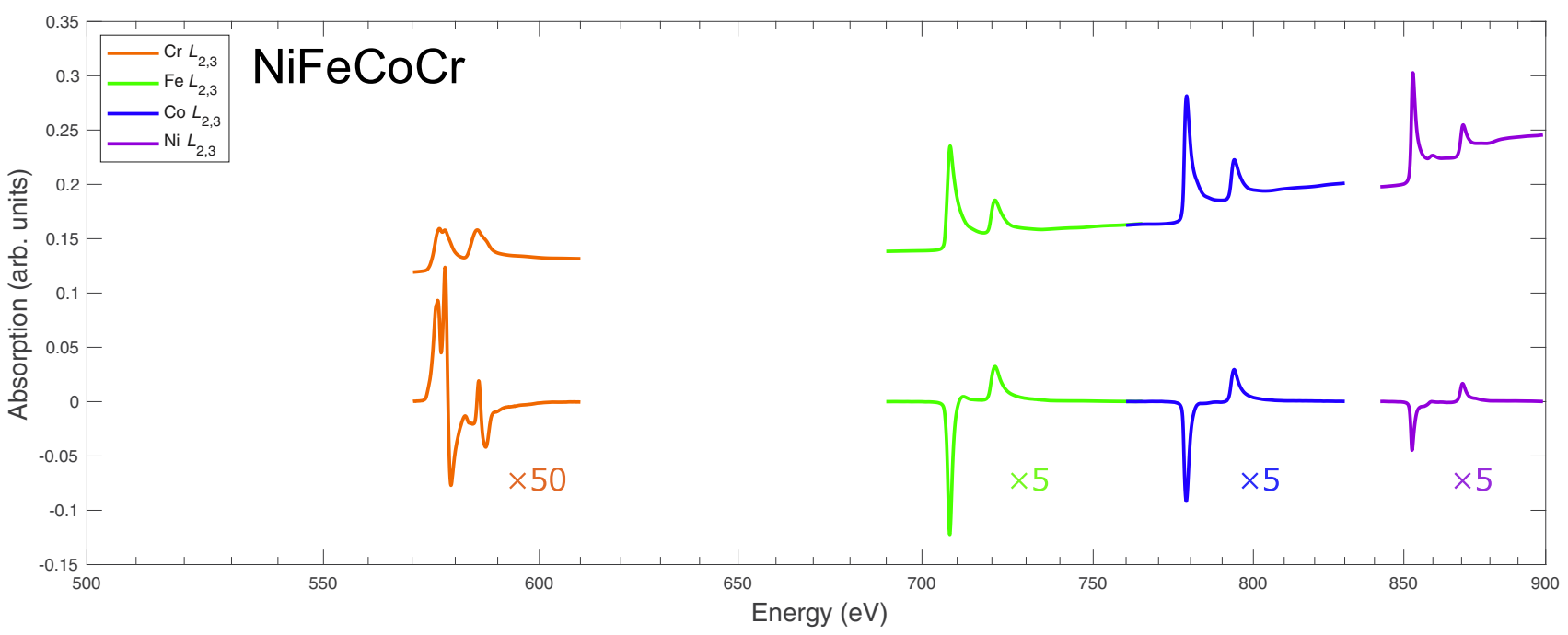

FIG. 2. XAS (top) and XMCD (bottom) spectra of (left to right) $\mathrm{Cr}, \mathrm{Fe}, \mathrm{Co}$, and $\mathrm{Ni}$ in (a) NiFeCo and (b) NiFeCoCr. The multiplication labels indicate the scaling of the XMCD signals. All of the XAS signals have been offset by a constant value of 0.1 for clarity. Note that the incident photon energy is plotted on a logarithmic scale (the 50-eV energy intervals get closer together with increasing energy) for clarity because at lower energies the core-level spin-orbit splitting of species with lower atomic number is smaller.

\section{DISCUSSION}

All of the experimental and calculated magnetic moments determined in this study are summarized in Table II. In all of the alloys measured here, both the sum rules and KKR-CPA calculations predict positive values for the orbital and spin moments of $\mathrm{Ni}, \mathrm{Fe}, \mathrm{Co}$, and $\mathrm{Mn}$, while the orbital moment is positive and the spin moment is negative for $\mathrm{Cr}$ (antiparallel spin and orbital moments are expected in $\mathrm{Cr}$ from Hund's rules). For every element in every alloy, the orbital moments are essentially quenched due to the (approximately) cubic symmetry; Co has the largest orbital sum rule moment, but it is never more than about $10 \%$ of the spin sum rule moment. Eriksson et al. predicted a large orbital moment for fcc Co [84].

In $\mathrm{NiFeCo}$, the experimentally determined bulk moments agree remarkably well with the KKR-CPA calculations. Given that $\mathrm{NiFeCo}$ is the least compositionally complex of the alloys investigated here and that all of the elements present have ferromagnetic couplings, this is hardly surprising. For $\mathrm{NiFeCo}, \mathrm{NiFeCoCr}$, and $\mathrm{NiFeCoCrPd}$, the Fe moments from the sum rules also agree remarkably well with the KKR-CPA calculations. However, the $\mathrm{Ni}$ and $\mathrm{Co}$ orbital and spin sum rule moments are significantly overestimated leading to a species-averaged moment that is larger than both the KKRCPA calculations and bulk measurements. In fact, compared to the KKR-CPA calculations the Ni spin sum rule moment is systematically overestimated by $30-50 \%$ in NiFeCo, NiFe$\mathrm{CoCr}$, and $\mathrm{NiFeCoCrPd}$, while the Co spin sum rule moment is systematically overestimated by $10-20 \%$ in $\mathrm{NiFeCo}$ and $\mathrm{NiFeCoCrPd}$. In $\mathrm{NiFeCoCrPd}$, the sum rule moments show reasonable agreement with the average KKR-CPA moments, but both are significantly larger than the bulk measurements.

The situation is much more complicated for $\mathrm{NiFeCoCr}$ and $\mathrm{NiFeCoCrMn}$, both of which have been reported to exhibit spin-glass behavior [26,28,29]. In $\mathrm{NiFeCoCr}$, the elementspecific sum rule and calculated moments are in reasonable 
(a)

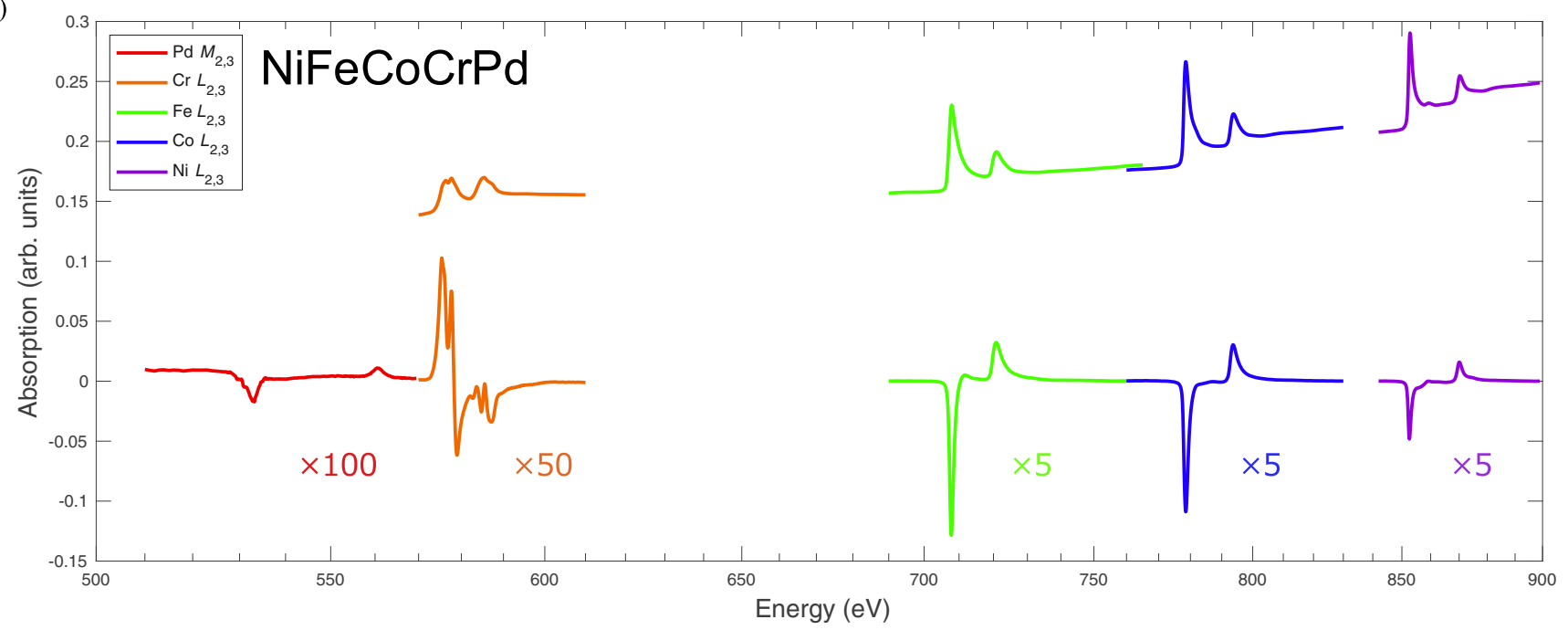

(b)

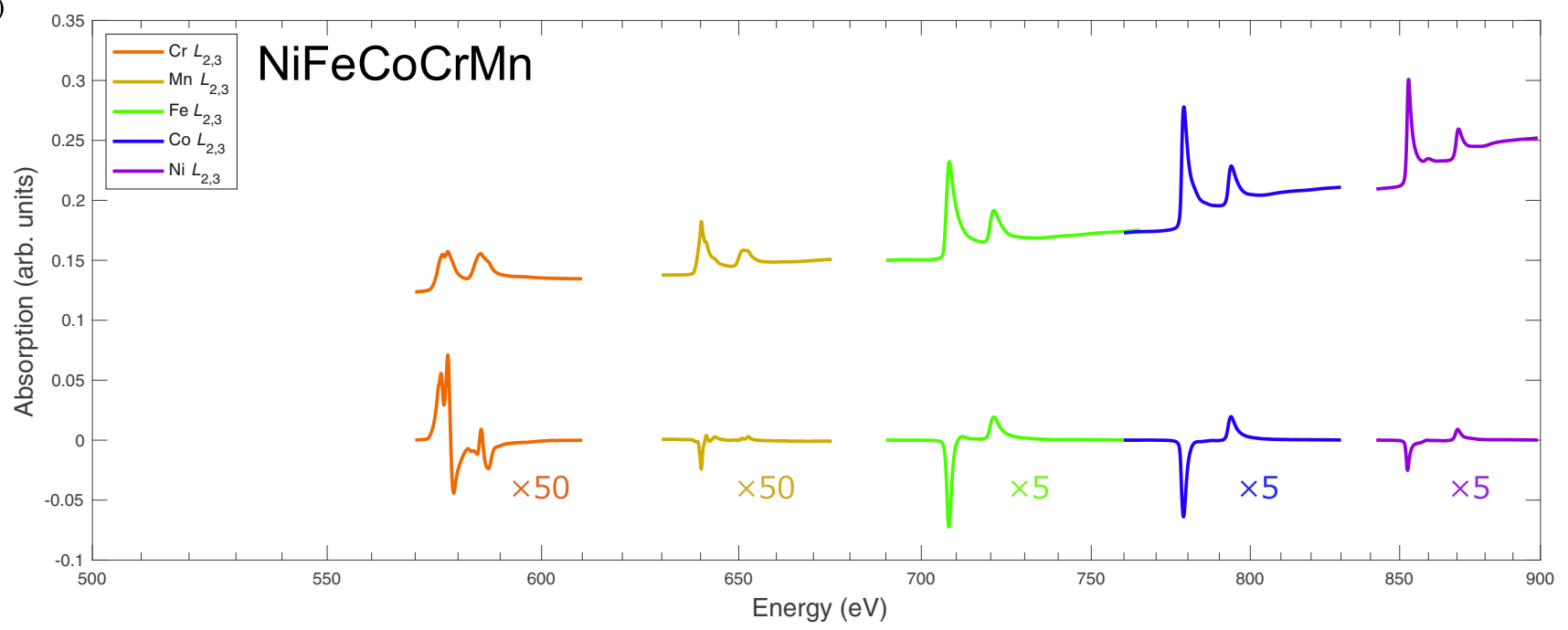

FIG. 3. XAS (top) and XMCD (bottom) spectra of (left to right) Pd, Cr, Mn, Fe, Co, and Ni in (a) NiFeCoCrPd and (b) NiFeCoCrMn. The multiplication labels indicate the scaling of the XMCD signals. All of the XAS signals have been offset by a constant value of 0.1 for clarity. Note that the incident photon energy is plotted on a logarithmic scale (the 50-eV energy intervals get closer together with increasing energy) for clarity because at lower energies the core-level spin-orbit splitting of species with lower atomic number is smaller.

agreement with each other, but the species-averaged moments are much larger than those determined by the SQUID and Compton measurements. It is worth noting that Jin et al. found a similar discrepancy between their experimental $\left(m^{\text {sat }}=0.24 \mu_{\mathrm{B}}\right)$ and calculated $\left(m^{\text {tot }}=0.66 \mu_{\mathrm{B}}\right.$ atom $\left.^{-1}\right)$ species-averaged total magnetic moment of $\mathrm{NiFeCoCr}$ to the one found here, which they suggested may well be indicative of a more complex, noncollinear, magnetic ground state than allowed by their KKR-CPA calculations, which were restricted to collinearity [26].

In $\mathrm{NiFeCoCrMn}$, the element-specific sum rule and calculated moments are in disagreement with each other, and the species-averaged moments are much larger than those determined by our SQUID measurements and those of Jin et al. [26]. In terms of the elemental moments, the largest discrepancy is seen for Mn; the KKR-CPA calculation predicts a large spin moment of $1.8 \mu_{\mathrm{B}}$ atom $^{-1}$, while the observed $\mathrm{XMCD}$ signal is extremely small, giving a spin sum rule moment less than $0.01 \mu_{\mathrm{B}}$ atom $^{-1}$. Again, this suggests that the KKR-CPA calculations employed here are not sophisticated enough to describe the real magnetic state of these two alloys. Indeed, Schneeweiss et al. [29] and $\mathrm{Mu}$ et al. [33] independently performed more sophisticated spin collinear supercell calculations and disordered local moment (DLM) KKR-CPA calculations, respectively, which both predict that in $\mathrm{NiFeCoCrMn}$ there are approximately equal populations of Mn atoms with large moments $\left(1.5 \mu_{\mathrm{B}}\right.$ atom $^{-1}$ to $2 \mu_{\mathrm{B}}$ atom $\left.^{-1}\right)$ aligned parallel $\left(\mathrm{Mn}^{\uparrow}\right)$ and antiparallel $\left(\mathrm{Mn}^{\downarrow}\right)$ to the spin quantization axis which would give us a species-averaged moment of $0.5 \mu_{\mathrm{B}}$ atom $^{-1}$ and a vanishing Mn site-averaged moment which is in much better agreement with the XMCD measurements. Interestingly, $\mathrm{Mu}$ et al. [34] also found an unconventional CPA ground state in NiCoMn which distinguishes two equally populated Mn CPA components with large but oppositely oriented spin moments and, using spin spiral calculations, they further demonstrated this calculated ground state is most energetically favorable in the presence of spin noncollinearity. The present XMCD measurements 
(a)

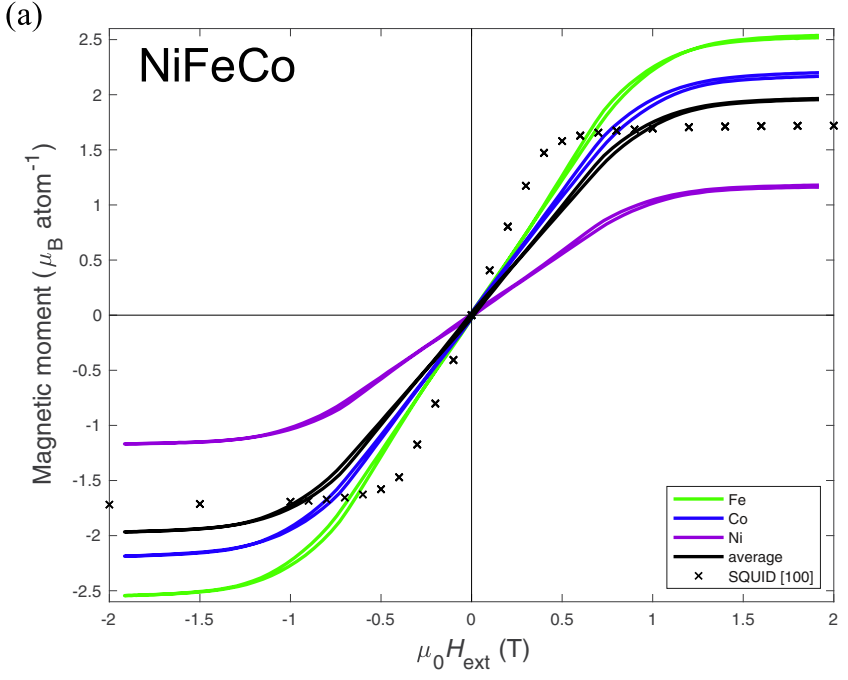

(c)

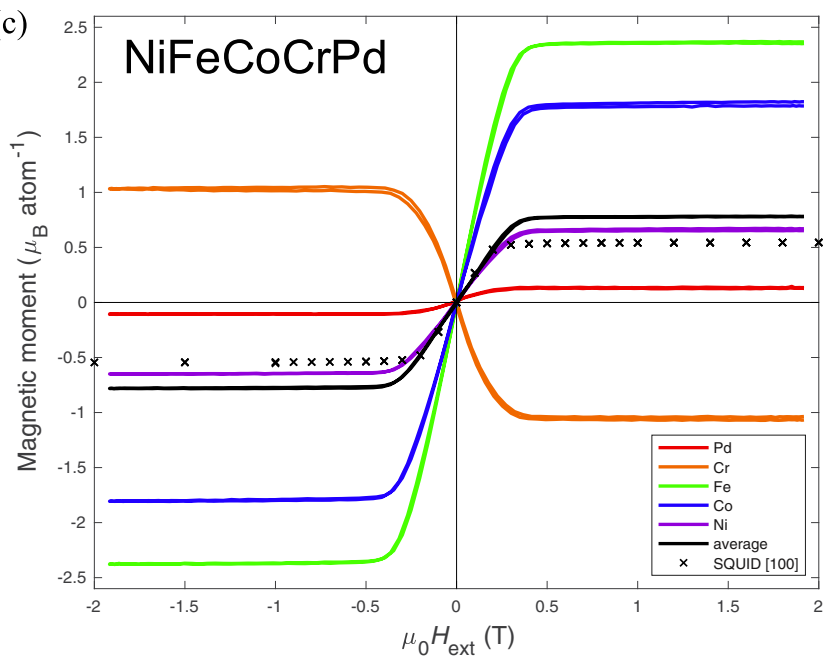

(b)

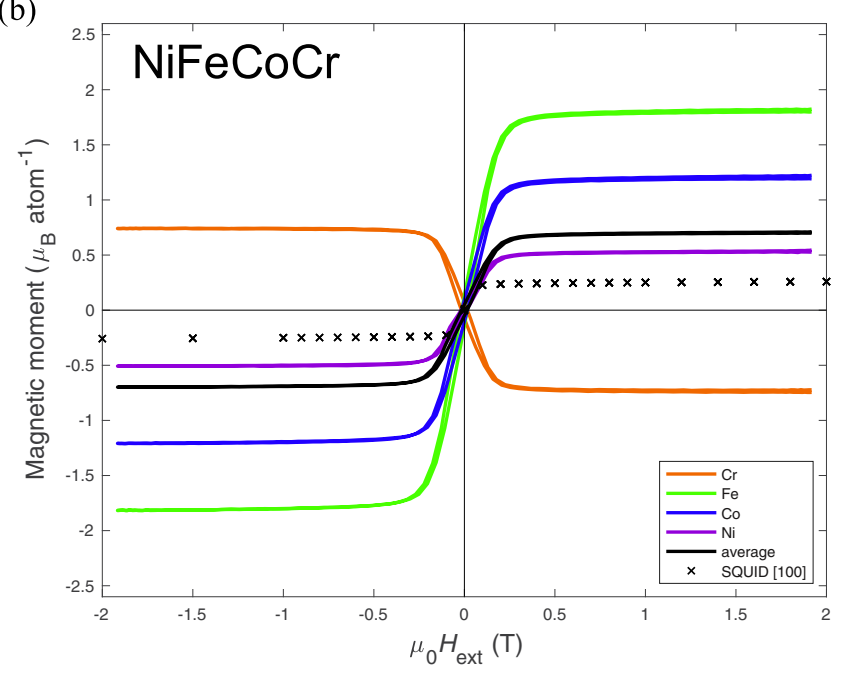

(d)

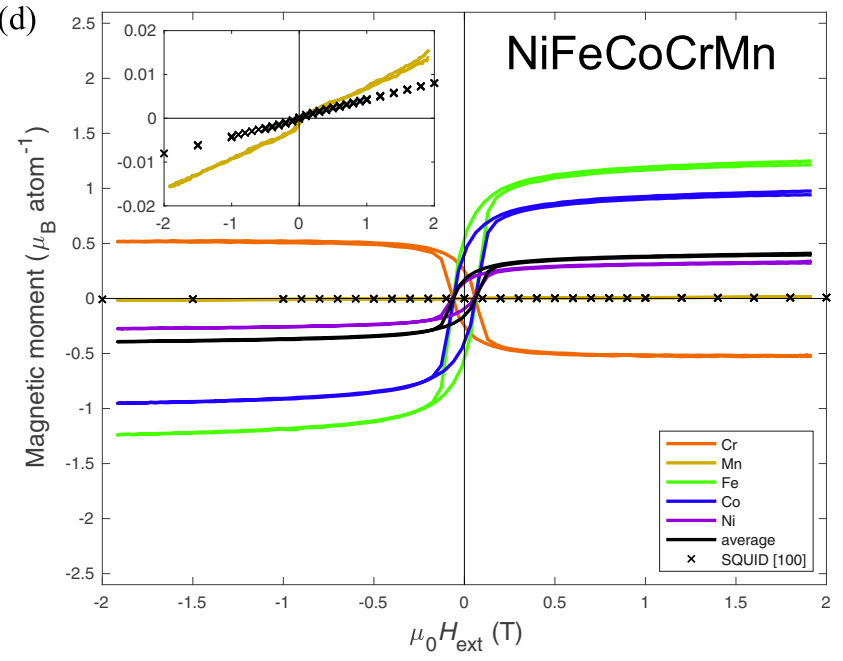

FIG. 4. Element-specific magnetization curves for (a) NiFeCo, (b) NiFeCoCr, (c) NiFeCoCrPd, and (d) NiFeCoCrMn determined by the XMCD measurements together with their respective magnetization curves determined by the SQUID measurements. The saturated magnetic moments for each atomic species have been scaled to the corresponding total moments determined by the orbital and spin sum rules (except for Pd where it was scaled to the total Pd $d$-electron moments from the KKR-CPA calculations) listed in Table II. The inset of (d) shows a close-up of the NiFeCoCrMn magnetization curve from the SQUID measurements together with the Mn magnetization curve from the XMCD measurements. The inset has the same axis labels and units as those of the main panel.

on $\mathrm{NiFeCoCrMn}$ provide the first strong experimental evidence for the existence of such a bizarre magnetic state, the prediction of which could be considered a triumph of the KKR-CPA-DLM theory.

In order to compare with the experimental MCPs, the calculated MCPs were normalized to the experimental spin moment. This procedure is not strictly valid given that the calculated moments (listed in Table II) are related to the exchange splitting of the majority and minority spin bands, which could change the Fermi surface size and topology, to both of which the shape of the MCP is sensitive [71]. Nevertheless, the calculations normalized to the experimental bulk spin moment provide an excellent description of the experimental data. It is well known that the LDA is a mean-field (Stoner) level theory which neglects spin fluctuations that can renormalize the magnetic moment [85]. In fact, it is not unusual to have the calculations predicting larger magnetic moments than experiment, and previous Compton scattering experiments [86] have required a similar rescaling of the profile area in order to agree with the experimental data, with the factor as large as 3 in UCoGe [87].

The species-averaged total and spin moments from the different experimental techniques and calculations are shown in Fig. 5 as a function of the concentration of antiferromagnetically coupled elements $(\mathrm{Cr}+\mathrm{Mn})$ in each alloy studied here, together with those of $\mathrm{NiCoCr}$ from Ref. [27]. The total and spin moments from the bulk measurements (SQUID and Compton, respectively) decrease approximately linearly with increasing $(\mathrm{Cr}+\mathrm{Mn})$ concentration. Compared with the bulk measurements, the sum rules systematically overestimate the average spin and, therefore, average total moments of each alloy, whereas the average orbital moments agree within the error bar. There may be significant errors in the spin sum rule moments [88]. The orbital and spin sum rules were originally formulated on the basis of a single ion in cubic symmetry, which has a well-defined number of $d$ electrons. 


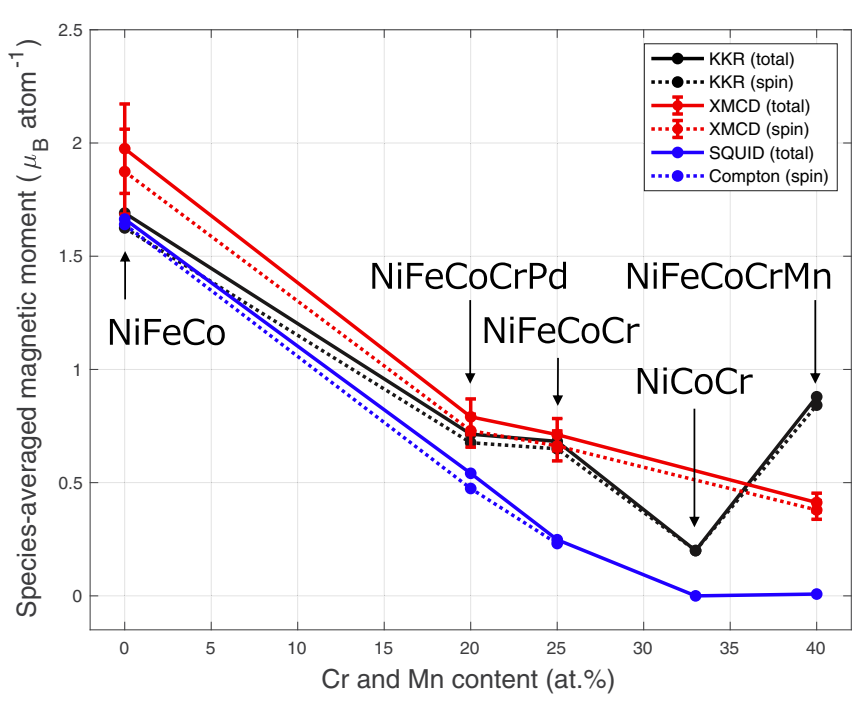

FIG. 5. Species-averaged total moments and spin moments as a function of the relative combined $\mathrm{Cr}$ and $\mathrm{Mn}$ concentration for $\mathrm{NiFeCo}(0$ at. $\% \mathrm{Cr}+\mathrm{Mn}), \mathrm{NiFeCoCr}(25$ at. $\% \mathrm{Cr}+\mathrm{Mn})$, NiFe$\mathrm{CoCrPd}(20$ at. $\% \mathrm{Cr}+\mathrm{Mn}$ ), and $\mathrm{NiFeCoCrMn}(40$ at. $\% \mathrm{Cr}+\mathrm{Mn}$ ) as determined by KKR-CPA calculations (black), the XMCD orbital and spin sum rules (red), and bulk (SQUID and Compton) measurements (blue). The experimental (SQUID) and calculated (KKR) total moments of $\mathrm{NiCoCr}$ (33 at. \% $\mathrm{Cr}+\mathrm{Mn}$ ) from Ref. [27] are also plotted. Solid and dotted lines are guides to the eye for the total moment and the spin moment, respectively. The orbital moment is given by the difference between the total moment and the spin moment. The orbital moment of $\mathrm{NiCoCr}$ has not been reported so we have set the calculated $m^{\text {tot }}=m^{\text {spin }}$, and magnetic Compton scattering was not performed on $\mathrm{NiCoCr}$ or $\mathrm{NiFeCoCrMn}$ so is not plotted. The error bars are statistical errors of one standard deviation. The error bars from the bulk measurements are approximately equal to the size of the points.

First-principles calculations indicate that $\left\langle T_{z}\right\rangle$ can reach values of $8.5 \%, 12 \%$, and $15 \%$ of $\left\langle S_{z}\right\rangle$ at the Fermi level for the $\mathrm{Fe}(001), \mathrm{Ni}(001)$, and $\mathrm{Co}(0001)$ surfaces, respectively $[89,90]$. It is worth noting that, through fits of the XAS spectra, Goering [59] determined that the spin sum rule moments of metallic $\mathrm{Fe}, \mathrm{Co}$, and $\mathrm{Ni}$ are overestimated by about $25 \%$, $35 \%$, and $45 \%$, respectively. Furthermore, calculations show that electron bands become flatter (narrower bandwidth) at the surface compared with the bulk due to the loss of near neighbors (which reduces the hopping integral), leading to enhanced spin and orbital magnetic moments [91-93].

At this point, it is worth considering that the KKR-CPA calculations employed here might actually be representative of the surface magnetic state. Indeed, Fig. 5 shows that the sum rule and KKR-CPA species-averaged moments show qualitative agreement over the whole series of alloys studied here, except for $\mathrm{NiFeCoCrMn}$ which can also be brought into agreement through more sophisticated calculations $[29,33]$. It is clear that the internal magnetic structures of the $\mathrm{Cr}$ - and Mn-containing alloys are much more complicated than the surface sensitive measurements suggest, and might be subject to strong spin fluctuations that suppress the bulk moments [27]. The differences between the experimental bulk and theoretical KKR-CPA moments might be due to short-range compositional order in these alloys [9,11-17], which is not considered in the single-site version of the KKR-CPA calculations used in this study (cluster versions of the CPA exist which can treat short-range compositional order, but such calculations are beyond the scope of this study). For example, first-principles calculations indicate that structural ordering of $\mathrm{Cr}$ in $\mathrm{NiFeCoCr}$ (with $\mathrm{Cr}$ atoms located on the corners of the cubic unit cell and randomized $\mathrm{Ni}, \mathrm{Fe}$, and Co atoms, i.e., the $\mathrm{L}_{2}$ structure) can relieve the frustrated magnetic interactions leading to a lower bulk total moment (due to a larger antiparallel Cr moment) [23]. Note that MCPs determined from supercell calculations of $\mathrm{NiFeCoCr}$ in the $\mathrm{L1}_{2}$ structure show much worse agreement with experiment.

Finally, although the alloys have been chemically etched and $\mathrm{Ar}$ ion sputtered to remove the surface oxide layer, which is evident as the $\mathrm{Ni}, \mathrm{Fe}$, and $\mathrm{Co} L_{2,3}$ edges do not appear to have significant oxide contributions in their spectra, the complete removal of $\mathrm{Cr}$ oxide is difficult. It is also worth considering the thermodynamic stability of transitionmetal oxides, as represented graphically in the Ellingham diagram [94] which plots the change in the Gibbs free energy as a function of temperature for the formation of various transition-metal oxides from their respective pure metals. The change in the Gibbs free energy of formation for $\mathrm{Cr}_{2} \mathrm{O}_{3}$ and $\mathrm{MnO}$ is much more negative than for $\mathrm{NiO}, \mathrm{CoO}$, and the $\mathrm{Fe}$ oxides $\left(\mathrm{FeO}, \mathrm{Fe}_{3} \mathrm{O}_{4}\right.$, and $\left.\mathrm{Fe}_{2} \mathrm{O}_{3}\right)$, meaning that $\mathrm{Cr}$ and $\mathrm{Mn}$ will always be preferentially oxidized by any residual oxygen in the UHV chamber. In all of the Cr-containing alloys, the $\mathrm{Cr}$ XAS spectra have split peak structures at the $L_{3}$ edge. This could be due to contributions of both metallic $\mathrm{Cr}$ [95] and $\mathrm{Cr}$ oxides [96]. If present, $\mathrm{Cr}_{2} \mathrm{O}_{3}$ is only likely to exist very near the surface and would naturally provide the observed corrosion resistance [97].

\section{CONCLUSION}

Magnetic-field-dependent synchrotron x-ray experiments with circularly polarized photons and bulk magnetometry measurements were performed on a set of medium( $\mathrm{NiFeCo}$ and $\mathrm{NiFeCoCr}$ ) and high-entropy $(\mathrm{NiFeCoCrPd}$ and $\mathrm{NiFeCoCrMn)} \mathrm{Cantor-Wu} \mathrm{alloys.} \mathrm{The} \mathrm{bulk} \mathrm{spin} \mathrm{momentum}$ densities probed by magnetic Compton scattering are remarkably isotropic and this is a consequence of the smearing of the electronic structure by the compositional disorder. The bulk spin moments are in good agreement with the total moments from bulk magnetometry measurements indicating that the orbital moments are essentially quenched due to the (approximately) cubic symmetry. Finite XMCD signals were recorded for every element in every alloy, indicating differences in the populations of the majority and minority spin states (implying finite magnetic moments), and revealed that the $\mathrm{Cr}$ spin moments in the Cr-containing alloys are unambiguously aligned antiparallel to the bulk total moment. In NiFeCoCrMn, the total Mn magnetic moment is almost zero which suggests from previous work that this may be due to an approximately equal number of measured $\mathrm{Mn}$ moments which are parallel and antiparallel to the external field. Significant discrepancies between the experimental bulk and surface moments have been observed, and these are not in complete agreement with many of the KKR-CPA calculated moments. There could be 
contributions from short-range ordering in these samples or more complex alignment of the moments, which the calculations do not consider. From this study, a picture of the magnetism of the Cantor-Wu alloys emerges in which their bulk magnetic moments are increasingly suppressed with increasing concentration of antiferromagnetically coupled elements in the solid solution while the surface magnetic moments remain largely oblivious to these suppression mechanisms.

Looking forwards, questions still remain about the nature of the (apparent lack of) magnetism in NiCoCr. In KKR-CPA calculations, the magnetism of $\mathrm{NiCoCr}_{x}$ decreases linearly with increasing $\mathrm{Cr}$ content as the quantum critical point $(x \approx$ 1 ) is approached, but decreases exponentially in bulk magnetometry measurements [27]. Therefore, it would be interesting to repeat the XMCD measurements on $\mathrm{NiCoCr}_{x}$ with various compositions encompassing the quantum critical point to determine whether there are detectable magnetic moments at the sample surface that are suppressed in the bulk, and to understand their variation with $\mathrm{Cr}$ content compared with the bulk moments on either side of the quantum critical point. Another avenue worth exploring would be to investigate the spin dynamics in the bulk of these alloys, especially in $\mathrm{NiCoCr}$,
$\mathrm{NiFeCoCr}$, and $\mathrm{NiFeCoCrMn}$ using spin-polarized neutron scattering or muon spin rotation and relaxation.

The research data are available from the University of Bristol Research Data Repository [98].

\section{ACKNOWLEDGMENTS}

The magnetic Compton scattering and soft $x$-ray absorption spectroscopy experiments were performed with the approval of the Japan Synchrotron Radiation Research Institute (JASRI), Proposals No. 2016B0131 and No. 2017B1243, respectively. D.A.L. gratefully acknowledges the financial support of the National Secretariat of Higher Education, Science, Technology and Innovation of Ecuador (SENESCYT). S.M., G.D.S., and G.M.S. acknowledge funding support by the Energy Dissipation and Defect Evolution (EDDE), an Energy Frontier Research Center funded by the US Department of Energy (DOE), Office of Science, Basic Energy Sciences under Contract No. DE-AC05-00OR22725. We gratefully acknowledge the financial support of the UK EPSRC (Grants No. EP/R029962/1, No. EP/L015544/1, and No. EP/S016465/1).
[1] B. Cantor, Entropy 16, 4749 (2014).

[2] B. Cantor, I. T. H. Chang, P. Knight, and A. J. B. Vincent, Mater. Sci. Eng. A 375-377, 213 (2004).

[3] T. K. Chen, T. T. Shun, J. W. Yeh, and M. S. Wong, Surf. Coat. Technol. 188-189, 193 (2004).

[4] C.-Y. Hsu, J.-W. Yeh, S.-K. Chen, and T.-T. Shun, Metall. Mater. Trans. A 35, 1465 (2004).

[5] J.-W. Yeh, S.-J. Lin, T.-S. Chin, J.-Y. Gan, S.-K. Chen, T.-T. Shun, C.-H. Tsau, and S.-Y. Chou, Metall. Mater. Trans. A 35, 2533 (2004).

[6] J.-W. Yeh, S.-K. Chen, S.-J. Lin, J.-Y. Gan, T.-S. Chin, T.-T. Shun, C.-H. Tsau, and S.-Y. Chang, Adv. Eng. Mater. 6, 299 (2004).

[7] M.-H. Tsai, Entropy 15, 5338 (2013).

[8] M.-H. Tsai and J.-W. Yeh, Mater. Res. Lett. 2, 107 (2014).

[9] Y. Zhang, T. T. Zuo, Z. Tang, M. C. Gao, K. A. Dahmen, P. K. Liaw, and Z. P. Lu, Prog. Mater. Sci. 61, 1 (2014).

[10] R. Kozak, A. Sologubenko, and W. Steurer, Z. Kristallogr. Cryst. Mater. 230, 55 (2014).

[11] E. J. Pickering and N. G. Jones, Int. Mater. Rev. 61, 183 (2016).

[12] D. B. Miracle and O. N. Senkov, Acta Mater. 122, 448 (2017).

[13] M. S. Lucas, G. B. Wilks, L. Mauger, J. A. Muñoz, O. N. Senkov, E. Michel, J. Horwath, S. L. Semiatin, M. B. Stone, D. L. Abernathy, and E. Karapetrova, Appl. Phys. Lett. 100, 251907 (2012).

[14] A. Tamm, A. Aabloo, M. Klintenberg, M. Stocks, and A. Caro, Acta Mater. 99, 307 (2015).

[15] F. X. Zhang, S. Zhao, K. Jin, H. Xue, G. Velisa, H. Bei, R. Huang, J. Y. P. Ko, D. C. Pagan, J. C. Neuefeind, W. J. Weber, and Y. Zhang, Phys. Rev. Lett. 118, 205501 (2017).

[16] Z. Dong and L. Vitos, Scr. Mater. 171, 78 (2019).

[17] R. Zhang, S. Zhao, J. Ding, Y. Chong, T. Jia, C. Ophus, M. Asta, R. O. Ritchie, and A. M. Minor, Nature 581, 283 (2020).
[18] B. Gludovatz, A. Hohenwarter, D. Catoor, E. H. Chang, E. P. George, and R. O. Ritchie, Science 345, 1153 (2014).

[19] O. N. Senkov, D. Isheim, D. N. Seidman, and A. L. Pilchak, Entropy 18, 102 (2016).

[20] T. Csanádi, E. Castle, M. J. Reece, and J. Dusza, Sci. Rep. 9 , 10200 (2019).

[21] P. J. Barron, A. W. Carruthers, J. W. Fellowes, N. G. Jones, H. Dawson, and E. J. Pickering, Scr. Mater. 176, 12 (2020).

[22] Z. Wu, H. Bei, F. Otto, G. M. Pharr, and E. P. George, Intermetallics 46, 131 (2014).

[23] C. Niu, A. J. Zaddach, A. A. Oni, X. Sang, J. W. Hurt, J. M. LeBeau, C. C. Koch, and D. L. Irving, Appl. Phys. Lett. 106, 161906 (2015).

[24] D. G. Pettifor, Mater. Sci. Technol. 4, 675 (1988).

[25] S. Guo, C. Ng, J. Lu, and C. T. Liu, J. Appl. Phys. 109, 103505 (2011).

[26] K. Jin, B. C. Sales, G. M. Stocks, G. D. Samolyuk, M. Daene, W. J. Weber, Y. Zhang, and H. Bei, Sci. Rep. 6, 20159 (2016).

[27] B. C. Sales, K. Jin, H. Bei, G. M. Stocks, G. D. Samolyuk, A. F. May, and M. A. McGuire, Sci. Rep. 6, 26179 (2016).

[28] Y.-F. Kao, S.-K. Chen, T.-J. Chen, P.-C. Chu, J.-W. Yeh, and S.-J. Lin, J. Alloys Compd. 509, 1607 (2011).

[29] O. Schneeweiss, M. Friák, M. Dudová, D. Holec, M. Šob, D. Kriegner, V. Holý, P. Beran, E. P. George, J. Neugebauer, and A. Dlouhý, Phys. Rev. B 96, 014437 (2017).

[30] M. S. Lucas, L. Mauger, J. A. Muñoz, Y. Xiao, A. O. Sheets, S. L. Semiatin, J. Horwath, and Z. Turgut, J. Appl. Phys. 109, 07E307 (2011).

[31] Y. Zhang, T. Zuo, Y. Cheng, and P. K. Liaw, Sci. Rep. 3, 1455 (2013).

[32] F. Tian, L. K. Varga, N. Chen, L. Delczeg, and L. Vitos, Phys. Rev. B 87, 075144 (2013).

[33] S. Mu, G. D. Samolyuk, S. Wimmer, M. C. Troparevsky, S. N. Khan, S. Mankovsky, H. Ebert, and G. M. Stocks, npj Comput. Mater. 5, 1 (2019). 
[34] S. Mu, J. Yin, G. D. Samolyuk, S. Wimmer, Z. Pei, M. Eisenbach, S. Mankovsky, H. Ebert, and G. M. Stocks, Phys. Rev. Materials 3, 014411 (2019).

[35] D. A. Lagos, Ph.D. thesis, University of Bristol, Bristol, United Kingdom, 2019.

[36] K. Jin, S. Mu, K. An, W. D. Porter, G. D. Samolyuk, G. M. Stocks, and H. Bei, Mater. Des. 117, 185 (2017).

[37] Y. Zhang, G. M. Stocks, K. Jin, C. Lu, H. Bei, B. C. Sales, L. Wang, L. K. Béland, R. E. Stoller, G. D. Samolyuk, M. Caro, A. Caro, and W. J. Weber, Nat. Commun. 6, 8736 (2015).

[38] G. D. Samolyuk, S. Mu, A. F. May, B. C. Sales, S. Wimmer, S. Mankovsky, H. Ebert, and G. M. Stocks, Phys. Rev. B 98, 165141 (2018).

[39] S. Mu, Z. Pei, X. Liu, and G. M. Stocks, J. Mater. Res. 33, 2857 (2018).

[40] H. C. Robarts, T. E. Millichamp, D. A. Lagos, J. Laverock, D. Billington, J. A. Duffy, D. O’Neill, S. R. Giblin, J. W. Taylor, G. Kontrym-Sznajd, M. Samsel-Czekała, H. Bei, S. Mu, G. D. Samolyuk, G. M. Stocks, and S. B. Dugdale, Phys. Rev. Lett. 124, 046402 (2020).

[41] N. E. Hussey, K. Takenaka, and H. Takagi, Philos. Mag. 84, 2847 (2004).

[42] D. Buterakos and S. Das Sarma, Phys. Rev. B 100, 235149 (2019).

[43] M. J. Cooper, Radiat. Phys. Chem. 50, 63 (1997).

[44] J. E. McCarthy, M. J. Cooper, V. Honkimäki, T. Tschentscher, P. Suortti, S. Gardelis, K. Hämäläinen, S. O. Manninen, and D. N. Timms, Nucl. Instrum. Methods Phys. Res. Sect. A 401, 463 (1997).

[45] T. Funk, A. Deb, S. J. George, H. Wang, and S. P. Cramer, Coord. Chem. Rev. 249, 3 (2005).

[46] B. T. Thole, P. Carra, F. Sette, and G. van der Laan, Phys. Rev. Lett. 68, 1943 (1992).

[47] P. Carra, B. T. Thole, M. Altarelli, and X. Wang, Phys. Rev. Lett. 70, 694 (1993).

[48] H. Bei and E. P. George, Acta Mater. 53, 69 (2005).

[49] M. J. Cooper, E. Zukowski, S. P. Collins, D. N. Timms, F. Itoh, and H. Sakurai, J. Phys.: Condens. Matter 4, L399 (1992).

[50] P. Carra, M. Fabrizio, G. Santoro, and B. T. Thole, Phys. Rev. B 53, R5994 (1996).

[51] T. Hara, K. Shirasawa, M. Takeuchi, T. Seike, Y. Saito, T. Muro, and H. Kitamura, Nucl. Instrum. Methods Phys. Res. Sect. A 498, 496 (2003).

[52] T. Hirono, H. Kimura, T. Muro, Y. Saitoh, and T. Ishikawa, J. Electron Spectrosc. Relat. Phenom. 144-147, 1097 (2005).

[53] T. Nakamura, T. Muro, F. Z. Guo, T. Matsushita, T. Wakita, T. Hirono, Y. Takeuchi, and K. Kobayashi, J. Electron Spectrosc. Relat. Phenom. 144-147, 1035 (2005).

[54] Y. Senba, H. Ohashi, Y. Kotani, T. Nakamura, T. Muro, T. Ohkochi, N. Tsuji, H. Kishimoto, T. Miura, M. Tanaka, M. Higashiyama, S. Takahashi, Y. Ishizawa, T. Matsushita, Y. Furukawa, T. Ohata, N. Nariyama, K. Takeshita, T. Kinoshita, A. Fujiwara, M. Takata, and S. Goto, AIP Conf. Proc. 1741, 030044 (2016).

[55] M. Abbate, J. B. Goedkoop, F. M. F. de Groot, M. Grioni, J. C. Fuggle, S. Hofmann, H. Petersen, and M. Sacchi, Surf. Interface Anal. 18, 65 (1992).

[56] J. Vogel and M. Sacchi, J. Electron Spectrosc. Relat. Phenom. 67, 181 (1994).
[57] B. H. Frazer, B. Gilbert, B. R. Sonderegger, and G. D. Stasio, Surf. Sci. 537, 161 (2003).

[58] C. T. Chen, Y. U. Idzerda, H.-J. Lin, N. V. Smith, G. Meigs, E. Chaban, G. H. Ho, E. Pellegrin, and F. Sette, Phys. Rev. Lett. 75, 152 (1995).

[59] E. Goering, Philos. Mag. 85, 2895 (2005).

[60] W. L. O'Brien and B. P. Tonner, Phys. Rev. B 50, 12672 (1994).

[61] E. Goering, A. Fuss, W. Weber, J. Will, and G. Schütz, J. Appl. Phys. 88, 5920 (2000).

[62] J. Korringa, Physica 13, 392 (1947).

[63] W. Kohn and N. Rostoker, Phys. Rev. 94, 1111 (1954).

[64] H. Ebert, D. Ködderitzsch, and J. Minár, Rep. Prog. Phys. 74, 096501 (2011).

[65] P. Soven, Phys. Rev. 156, 809 (1967).

[66] B. L. Gyorffy, Phys. Rev. B 5, 2382 (1972).

[67] J. S. Faulkner and G. M. Stocks, Phys. Rev. B 21, 3222 (1980).

[68] H. Ebert, The Munich SPR-KKR package, version 7 (2017).

[69] U. Dahlborg, J. Cornide, M. Calvo-Dahlborg, T. C. Hansen, A. Fitch, Z. Leong, S. Chambreland, and R. Goodall, J. Alloys Compd. 681, 330 (2016).

[70] S. H. Vosko, L. Wilk, and M. Nusair, Can. J. Phys. 58, 1200 (1980).

[71] M. A. G. Dixon, J. A. Duffy, S. Gardelis, J. E. McCarthy, M. J. Cooper, S. B. Dugdale, T. Jarlborg, and D. N. Timms, J. Phys.: Condens. Matter 10, 2759 (1998).

[72] Y. Kubo and S. Asano, Phys. Rev. B 42, 4431 (1990).

[73] D. N. Timms, A. Brahmia, M. J. Cooper, S. P. Collins, S. Hamouda, D. Laundy, C. Kilbourne, and M.-C. S. Lager, J. Phys.: Condens. Matter 2, 3427 (1990).

[74] V. Sundararajan and D. G. Kanhere, J. Phys.: Condens. Matter 3, 3311 (1991).

[75] P. E. Mijnarends, Physica 63, 235 (1973).

[76] D. Benea, J. Minár, L. Chioncel, S. Mankovsky, and H. Ebert, Phys. Rev. B 85, 085109 (2012).

[77] L. Chioncel, D. Benea, S. Mankovsky, H. Ebert, and J. Minár, Phys. Rev. B 90, 184426 (2014).

[78] L. Chioncel, D. Benea, H. Ebert, I. Di Marco, and J. Minár, Phys. Rev. B 89, 094425 (2014).

[79] L. Lam and P. M. Platzman, Phys. Rev. B 9, 5122 (1974).

[80] R. Nakajima, J. Stöhr, and Y. U. Idzerda, Phys. Rev. B 59, 6421 (1999).

[81] J. P. Crocombette, M. Pollak, F. Jollet, N. Thromat, and M. Gautier-Soyer, Phys. Rev. B 52, 3143 (1995).

[82] A. D. Alvarenga, F. Garcia, L. C. Sampaio, C. Giles, F. Yokaichiya, C. A. Achete, R. A. Simão, and A. P. Guimarães, J. Magn. Magn. Mater. 233, 74 (2001).

[83] A. D. Alvarenga, F. Garcia, W. D. Brewer, M. Gruyters, M. Gierlings, M. S. Reis, P. Panissod, L. C. Sampaio, and A. P. Guimarães, J. Magn. Magn. Mater. 242-245, 958 (2002).

[84] O. Eriksson, B. Johansson, R. C. Albers, A. M. Boring, and M. S. S. Brooks, Phys. Rev. B 42, 2707(R) (1990).

[85] A. Aguayo, I. I. Mazin, and D. J. Singh, Phys. Rev. Lett. 92 , 147201 (2004).

[86] T. D. Haynes, I. Maskery, M. W. Butchers, J. A. Duffy, J. W. Taylor, S. R. Giblin, C. Utfeld, J. Laverock, S. B. Dugdale, Y. Sakurai, M. Itou, C. Pfleiderer, M. Hirschberger, A. Neubauer, W. Duncan, and F. M. Grosche, Phys. Rev. B 85, 115137 (2012). 
[87] M. W. Butchers, J. A. Duffy, J. W. Taylor, S. R. Giblin, S. B. Dugdale, C. Stock, P. H. Tobash, E. D. Bauer, and C. Paulsen, Phys. Rev. B 92, 121107(R) (2015).

[88] C. Piamonteze, P. Miedema, and F. M. F. de Groot, Phys. Rev. B 80, 184410 (2009).

[89] R. Wu and A. J. Freeman, Phys. Rev. Lett. 73, 1994 (1994).

[90] R. Wu, D. Wang, and A. J. Freeman, J. Magn. Magn. Mater. 132, 103 (1994).

[91] O. Eriksson, G. W. Fernando, R. C. Albers, and A. M. Boring, Solid State Commun. 78, 801 (1991).

[92] O. Eriksson, A. M. Boring, R. C. Albers, G. W. Fernando, and B. R. Cooper, Phys. Rev. B 45, 2868 (1992).
[93] S. Keshavarz, Y. O. Kvashnin, I. Di Marco, A. Delin, M. I. Katsnelson, A. I. Lichtenstein, and O. Eriksson, Phys. Rev. B 92, 165129 (2015).

[94] H. J. T. Ellingham, J. Soc. Chem. Ind. 63, 125 (1944).

[95] M. A. Tomaz, W. J. Antel, W. L. O’Brien, and G. R. Harp, Phys. Rev. B 55, 3716 (1997).

[96] É. Gaudry, P. Sainctavit, F. Juillot, F. Bondioli, P. Ohresser, and I. Letard, Phys. Chem. Miner. 32, 710 (2006).

[97] Y. Qiu, S. Thomas, M. A. Gibson, H. L. Fraser, and N. Birbilis, npj Mater. Degrad. 1, 15 (2017).

[98] S. B. Dugdale, https://doi.org/10.5523/bris. 4ji1p911bh6t20mcg6zgwwqwv. 